\title{
Examples of Nonsingular Irreducible Curves Which Give Reducible Singular Points of $\operatorname{red}\left(\mathrm{H}_{d, g}\right)$
}

by

\author{
Mutsumi AMASAKI*
}

\section{Introduction}

The open subscheme $\mathrm{H}_{d, g}$ of $\mathrm{Hilb}\left(\mathbb{P}^{3}\right)$ which consists of points corresponding to nonsingular irreducible curves of degree $d$ and genus $g$ has more than one irreducible components in many cases. If one proceeds further to care about its connected components, he will necessarily encounter the problem whether $\operatorname{red}\left(\mathrm{H}_{d, g}\right)$ is irreducible at the point corresponding to a given curve or not. But even the examples of such reducible singular points of $\operatorname{red}\left(\mathrm{H}_{d, g}\right)$ do not seem to be known well, except that J. Harris in [6; p. 93] mentioned the existence of nondegenerate nonsingular irreducible curves in $\mathbb{P}^{n}(n \geqq 4)$ whose Hilbert points lie on more than one irreducible components of $\operatorname{Hilb}\left(\mathbf{P}^{n}\right)$. These curves are on the cone over a nonsingular rational curve of degree $n-1$ in $\mathbb{P}^{n-1}$ and the projection of them to $\mathbb{P}^{3}$ provides the examples of curves in $\mathbb{P}^{3}$ which have the same character, if the degree is sufficiently small as compared with the genus. For instance, when $n=4$ and $\hat{C}$ is a nonsingular irreducible curve belonging to the linear system $|m h+r|(h$ : a hyperplane section, $r$ : a line of ruling) on the blowing up of the cone over a twisted cubic curve in $\mathbb{P}^{3}$ with center its vertex, the curve $X$ obtained by projecting $C$ (the isomorphic image of $\hat{C}$ under the blowing up) to $\mathbb{P}^{3}$ from a general point corresponds to a point of the intersection of two nonreduced irreducible components of $\mathrm{Hilb}\left(\mathbf{P}^{3}\right)$ for $m \gg 0$ (see [4; Theorem 3.1] and [5; Proposition B.2]). The basic sequence (cf. [2; Definition 1.4])

Communicated by S. Nakano, October 4, 1984.

* Research Institute for Mathematical Sciences, Kyoto University, Kyoto 606, Japan 
of $X$ is $\left(3 ; m+1,(m+2)^{2} ; 2 m+1\right)$, and under the deformation given in this way, $X$ is deformed on the one hand into curves having the same basic sequence and on the other hand into curves with basic sequence $\left(3 ;(m+2)^{3} ; m+2,2 m\right)$ (see Example A. 5 in the appendix of this paper together with Notation and Terminology 6). One of the features of this example is that the minimal degree of the surfaces containing the curve does not change in the deformation.

We will give other kinds of examples with completely different methods. In Section 3 we show the existence of a nonsingular irreducible arithmetically Buchsbaum curve $X_{1}$ with basic sequence (a; $\left.(a+2)^{a-1}, a+3 ; a+2\right) \quad(a \geqq 4)$ which can be deformed flatly into projectively Cohen-Macaulay curves in two different ways: one by deforming the homogeneous ideal $I_{X_{1}} \subset R:=k\left[x_{1}, x_{2}, x_{3}, x_{4}\right]$ which defines $X_{1}$ and the other by deforming the graded $R$-module $\mathrm{H}_{*}^{0}\left(\mathcal{O}_{x_{1}}\right)$ flatly. The basic sequences of the projectively Cohen-Macaulay curves obtained through these deformations are $\left(a ;(a+2)^{a}\right)$ and $\left(a+1 ;(a+1)^{3}\right.$, $\left.(a+2)^{a-3}, a+3\right)$ respectively. In Section 2 it is proved that there is a nonsingular irreducible curve $X_{2}$ with $\mathrm{H}_{*}^{1}\left(\mathscr{I}_{X_{2}}\right) \cong R[-a] /\left(x_{1}, x_{2}, A, B\right)$

(where $A, B$ are relatively prime homogeneous polynomials of degree 2 of $\left.k\left[x_{3}, x_{4}\right]\right)$ and having the basic sequence $\left(a ; a+2,(a+3)^{a-1} ; a+4\right)$ or $\left(a ;(a+2)^{a-3},(a+3)^{3} ; a+4\right)$ which can be deformed flatly at least in two different directions, namely one into a projectively CohenMacaulay curve and the other into an arithmetically Buchsbaum curve $X_{2}^{\prime}$ with $\mathrm{H}_{*}^{1}\left(\mathscr{I}_{X_{2}^{\prime}}\right)=k^{2}[-(a+1)]$. The latter cannot be induced either by the deformation of the ideal $I_{X_{2}}$ nor by that of $\mathrm{H}_{*}^{0}\left(\mathcal{O}_{X_{2}}\right)$. In the proof of the existence of the curves $X_{1}$ and $X_{2}$ we have used the technique of liaison to construct the desired curve from a simple and familiar one. It should be noted that in all the cases treated here the surface of degree $a$ which contains the curve in question is smooth, and in the deformation, the minimal degree of the surfaces containing the curve varies in one direction and does not in the other. The interested reader will be able to find many other examples by our method, if he wishes.

\section{Notation and Terminnology}

1. $k$ denotes an algebraically closed field of characteristic zero 
throughout this paper and we set $R:=k\left[x_{1}, x_{2}, x_{3}, x_{4}\right], \mathfrak{m}:=\left(x_{1}, x_{2}, x_{3}, x_{4}\right) R$, where $x_{1}, x_{2}, x_{3}, x_{4}$ are indeterminates over $k$.

2. $\mathbf{P}^{n}$ denotes the projective space of dimension $n$ over $k$.

3. We use the word 'curve' to mean an equidimensional complete scheme over $k$ of dimension one without any embedded points.

4. The notation of [2] will be used freely.

5. The ideal sheaf of a curve $X$ in $\mathbb{P}^{3}$ is denoted by $\mathscr{I}_{X}$ and $I_{X}=\bigoplus_{\nu \geq 0} I_{X, \nu}$ denotes the homogeneous ideal $\mathrm{H}_{*}^{0}\left(\mathbb{P}^{3}, \mathscr{I}_{X}\right)$ in $\mathrm{H}_{*}^{0}\left(\mathbb{P}^{3}, \mathcal{O}_{\mathbf{P}^{3}}\right)$ $=R$, where $I_{X, \nu}:=\mathrm{H}^{0}\left(\mathbb{P}^{3}, \mathscr{I}_{X}(\nu)\right)$.

6 . The sequence of integers $n, \cdots, n$ ( $m$ times) will often be denoted simply by $n^{m}$.

\section{§1. Some Remarks on the Basic Sequence of an Integral Curve}

Let $X$ be a curve in $\mathbf{P}^{3}$. We will denote by $B(X)$ the basic sequence $\left(a ; n_{1}, \ldots, n_{a} ; n_{a+1}, \ldots, n_{a+b}\right)$ of $X$ (see Definition 1.4 of [2], where the Greek letter $\nu$ is used instead of $n)$. Sometimes the symbol $\tilde{n}^{1}$ (resp. $\left.\bar{n}^{2}\right)$ is used to mean the sequence $\left(n_{1}, \ldots, n_{a}\right)\left(\operatorname{resp} .\left(n_{a+1}, \ldots\right.\right.$, $\left.n_{a+b}\right)$ ) for convenience sake. In this paper we say that an increasing sequence of integers $\left(z_{i}\right) i \geqq 1$ is connected if the difference $z_{i+1}-z_{i}$ is zero or one for all $\mathrm{i} \geqq 1$.

Lemma 1.1. Let $f$ be an irreducible polynomial of $R$ and let $s_{1}, s_{2}$, $t_{1}, t_{2}$ be algebraically independent elements over $R$. Then $\tilde{f}=f\left(x_{1}, x_{2}, s_{1} x_{1}+\right.$ $\left.s_{2} x_{2}, t_{1} x_{1}+t_{2} x_{2}\right)$ is irreducible as a polynomial of $Q:=k\left[s_{1}, s_{2}, t_{1}, t_{2}, x_{1}, x_{2}\right]$.

Proof. It is enough to show that the scheme $\operatorname{Spec}(Q / \tilde{f Q})$ is integral. In fact the open subscheme $\operatorname{Spec}(Q / \tilde{f} Q) \backslash \operatorname{Spec}\left(Q /\left(x_{1}, x_{2}\right) Q\right)$ is the union of two irreducible subschemes which are isomorphic respectively to

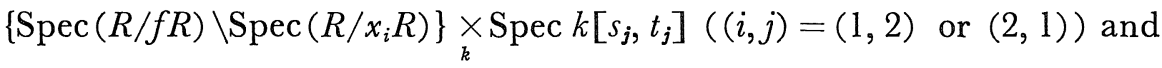
they have a Zariski open set in common, therefore the one codi$\begin{array}{ll}\text { mensional scheme } \operatorname{Spec}(Q / \tilde{f Q}) \text { is integral. } & \text { Q. E. D. }\end{array}$

Corollary 1.2. Let $\left(a ; n_{1}, \ldots, n_{a} ; n_{a+1}, \ldots, n_{a+b}\right)$ be the basic sequence of an integral curve $X$ in $\mathbf{P}^{3}$. Then $n_{1}, \ldots, n_{a}$ is a connected sequence of integers. 
Proof. First of all we may and do assume that the variables $x_{1}, x_{2}, x_{3}, x_{4}$ of $R$ are chosen sufficiently generally so that the argument in $[2 ; \S 1]$ may go well and we set $I:=I_{X}$. Recall that $\left(a ; n_{1}, \ldots, n_{a}\right)$ is defined as the sequence of the degrees of homogeneous polynomials $\bar{f}_{0}, \bar{f}_{1}, \ldots, \bar{f}_{a} \in k\left[x_{1}, x_{2}\right]$ satisfying $\bar{I}=I\left(\bmod \left(x_{3}, x_{4}\right)\right)=\bar{f}_{0} k\left[x_{1}, x_{2}\right] \oplus \bigoplus_{i=1}^{a} \bar{f}_{i} k\left[x_{2}\right]$. This definition can be restated as follows. Let $s_{1}, s_{2}, t_{1}, t_{2}$ be algebraically independent elements over $R, K$ the field $k\left(s_{1}, s_{2}, t_{1}, t_{2}\right)$ and put $I^{\prime}$ $=I K\left[x_{1}, x_{2}, x_{3}, x_{4}\right]$. Then $\left(a ; n_{1}, \ldots, n_{a}\right)$ is the sequence of the degrees of the homogeneous polynomials $\tilde{f}_{0}, \tilde{f}_{1}, \ldots, \tilde{f}_{a} \in K\left[x_{1}, x_{2}\right]$ such that

$$
\begin{aligned}
\tilde{I}: & =I^{\prime}\left(\bmod \left(x_{3}-s_{1} x_{1}-s_{2} x_{2}, x_{4}-t_{1} x_{1}-t_{2} x_{2}\right)\right) \\
& =\left\{\tilde{f}=f\left(x_{1}, x_{2}, s_{1} x_{1}+s_{2} x_{2}, t_{1} x_{1}+t_{2} x_{2}\right) \mid f \in I^{\prime}\right\} \\
& =\tilde{f}_{0} K\left[x_{1}, x_{2}\right] \oplus \bigoplus_{i=1}^{a} \tilde{f}_{i} K\left[x_{2}\right] \subset K\left[x_{1}, x_{2}\right] .
\end{aligned}
$$

Now let $\left[\begin{array}{c}\tilde{U}_{01} \\ \tilde{U}_{1}\end{array}\right]$ be the matrix of relations among $\tilde{f}_{0}, \tilde{f}_{1}, \ldots, \tilde{f}_{a}$ computed by $\left[1\right.$; Theorem 1.6] and suppose $n_{1}=\ldots=n_{u_{1}}<n_{u_{1}+1}=\ldots=n_{u_{1}+u_{2}}<$ $n_{u_{1}+u_{2}+1}=\ldots<n_{u_{1}+u_{2}+\cdots u_{r-1}+1}=\ldots=n_{u_{1}+u_{2}+\cdots+u_{r}}=n_{a}$. Since $X$ is integral by hypothesis, all elements of $I_{a}$ are irreducible, so $\tilde{I}_{a}$ contains an irreducible polynomial by Lemma 1.1, which implies, together with the formula

$$
\tilde{f}_{i}=(-1)^{i} \operatorname{det}\left[\begin{array}{c}
\tilde{U}_{01} \\
\tilde{U}_{1}
\end{array}\right]\left({ }^{i}\right)(0 \leqq i \leqq a)
$$

and the form of $\Delta\left(\left[\begin{array}{c}\tilde{U}_{01} \\ \tilde{U}_{1}\end{array}\right]\right)$ that

$$
\operatorname{rank}_{K}\left[\begin{array}{c}
\tilde{U}_{01} \\
\tilde{U}_{1}
\end{array}\right] \quad\left(\bmod \left(x_{1}, x_{2}\right)\right) \geqq r-1
$$

(cf. [4; Proposition 2.1]). Thus the sequence $n_{1}, \ldots, n_{a}$ must be connected.

Q. E. D.

One finds the following fact as a corollary to this proof.

Corollary 1.3. Let the notation be as in the preceeding corollary and let $\lambda_{2}=\left[\begin{array}{lll}U_{01} & U_{02} & 0 \\ U_{1} & U_{2} & U_{4} \\ U_{21} & U_{3} & U_{5}\end{array}\right]$ be the matrix of relations among the generators of $I=\mathrm{H}_{*}^{0}\left(\mathscr{I}_{X}\right)$ described in $\left[2 ;\right.$ Proposition 1.3]. Then $\operatorname{rank}_{k}\left[\begin{array}{l}U_{01} \\ U_{1}\end{array}\right](\bmod m)$ $\geqq r-1$, where $r=\#\left\{n_{1}, n_{2}, \ldots, n_{a}\right\}$. 
Proof. In the formula (1.2.1), we may replace $K$ by the ring $k\left[s_{1}, s_{2}, t_{1}, t_{2}\right]_{h}$ and $I^{\prime}$ by $I k\left[s_{1}, s_{2}, t_{1}, t_{2}\right]_{h}$, where $h$ is a polynomial of $k\left[s_{1}, s_{2}, t_{1}, t_{2}\right]$ such that $h \tilde{f}_{i} \in k\left[s_{1}, s_{2}, t_{1}, t_{2}, x_{1}, x_{2}\right]$ for all $0 \leqq i \leqq a$. Then the entries of $\tilde{U}_{01}, \tilde{U}_{1}$ are in $k\left[s_{1}, s_{2}, t_{1}, t_{2}, x_{1}, x_{2}\right]_{h}$ and the matrix of relations $\left[\begin{array}{l}U_{01} \\ U_{1}\end{array}\right]\left(\bmod \left(x_{3}, x_{4}\right)\right)$ among $\bar{f}_{0}, \bar{f}_{1}, \ldots, \bar{f}_{a}$ is induced from $\left[\begin{array}{c}\tilde{U}_{01} \\ \tilde{U}_{1}\end{array}\right]$ by substituting $(0,0,0,0)$ for $\left(s_{1}, s_{2}, t_{1}, t_{2}\right)$. Since $x_{3}, x_{4}$ were chosen sufficiently generally, the assertion follows from the last part of the proof of the preceding corollary.

Q.E.D.

The next results are all derived from the condition length $\mathrm{h}_{R}$ $\left(\right.$ Coker $\left.\left({ }^{t} \lambda_{3}\right)\right)<\infty \quad\left(\right.$ cf. $\left[1 ;(3.5 .5)^{\prime}\right]$ and $\left.[2 ;(2.1 .1)]\right)$. They are useful in certain restricted cases to compute the basic sequence of a given nonsingular irreducible curve. We will continue to use the notation $X, I$ and $\left(a ; n_{1}, \ldots, n_{a} ; n_{a+1}, \ldots, n_{a+b}\right)$ with the assumption that $X$ is integral.

Lemma 1.4. With the notation above, suppose $n_{1}=n_{2}$ and $n_{i}=n_{1}+i-2$ for $3 \leqq i \leqq a$. Put $j_{0}=\max \left\{j \mid n_{a+j}<n_{1}+a-2\right\}$. Then the sequence $n_{a+1}, \ldots$, $n_{a+j_{0}}$ is connected and

$$
\operatorname{rank}_{k}\left(\left[{ }^{t} U_{3}{ }^{t} U_{5}\right]\left(j_{0}+1, \ldots, b\right)\right)(\bmod \mathfrak{m}) \geqq q \text {, where } q=\#\left\{n_{a+1}, \ldots, n_{a+j_{0}}\right\} \text { 。 }
$$

Proof. Since the degrees of the entries of $\lambda_{2}$ are determined as stated in [1; Corollary 3.5], if the assertion were not true, one would have for some $p\left(1 \leqq p \leqq j_{0}\right)$

$$
U_{3}=\left[\begin{array}{l}
U_{3}^{\prime} \\
0
\end{array}\right], \quad U_{5}=\left[\begin{array}{l}
U_{5}^{\prime} \\
0
\end{array} *\right]
$$

with $p \times p$ matrices $U_{3}^{\prime}, U_{5}^{\prime}$ such that the entries of $U_{3}^{\prime}-x_{1} 1_{p}$ and $U_{5}^{\prime}-x_{2} 1_{p}$ are in $k(2)$. But this leads to a contradiction in the following

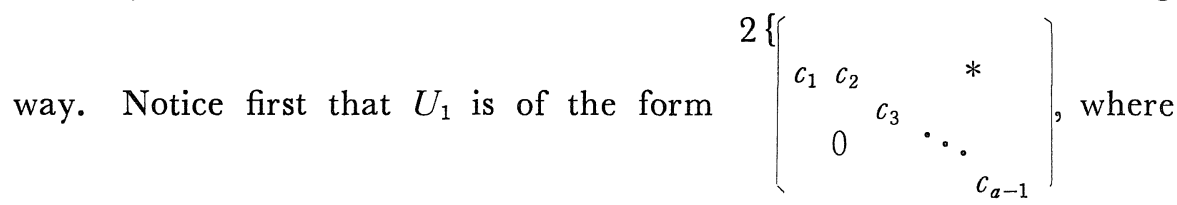
$c_{i} \in k \quad(1 \leqq i \leqq a-1)$ satisfy $c_{1} c_{3} \ldots c_{a-1} \neq 0$ or $c_{2} c_{3} \ldots c_{a-1} \neq 0$ by Corollary 1.3 , and recall the direct sum (1.4.1) $\quad R^{p}={ }^{t} U_{3}^{\prime} k(0)^{p} \oplus^{t} U_{5}^{\prime} k(1)^{p} \oplus k(2)^{p} \quad$ (cf. [1; Remark 4.1]). 
Let $h_{i}$ (resp. $l_{i}$ ) denote the $\mathrm{i}$-th row of

$$
U_{4}(p+1, \ldots, b)\left(\text { resp. } U_{2}(p+1, \ldots, b)\right)
$$

for $1 \leqq i \leqq a$. Then it follows from [2; Lemma 1.6] that $h_{a}=0$, for the degrees of the components of $h_{a}$ must be nonpositive by the choice of $j_{0}$. Starting from this fact we can prove the following:

Claim. $h_{i}=l_{i}=0$ for $3 \leqq i \leqq a$ and $c_{1} h_{1}+c_{2} h_{2}=0$.

Proof of Claim. Put $\lambda_{3}^{\prime}=\lambda_{3}(p+1, \ldots, b)=\left[\begin{array}{c}-h_{1} \\ \vdots \\ -h_{a} \\ -U_{5}^{\prime} \\ 0 \\ -U_{3}^{\prime} \\ 0\end{array}\right]$.

Suppose $h_{a}=\ldots=h_{i}=0$ for $3<i \leqq a$, then one finds by the equation $\lambda_{2} \lambda_{3}^{\prime}=0$ that $-c_{i-1} h_{i-1}-l_{i} U_{5}^{\prime}=0$, namely ${ }^{t} U_{5}^{\prime}\left(-{ }^{t} l_{i}\right)-c_{i-1}{ }^{t} h_{i-1}=0$, which implies by (1.4.1) that $l_{i}=h_{i-1}=0$. Thus $h_{i}=l_{i+1}=0$ for $3 \leqq i \leqq a$ by induction and finally we obtain $c_{1} h_{1}+c_{2} h_{2}=l_{3}=0$ analogously.

Now we go back to the proof of Lemma 1.4. The Claim and the formulae (2.3.5), (2.3.6) of [2] imply that

$$
\operatorname{Im}^{R}\left(\left({ }^{t} \lambda_{3}\right)(p+1, \ldots, b)\right)={ }^{t} U_{3}^{\prime} k(0)^{p} \oplus{ }^{t} U_{5}^{\prime} k(1)^{p} \oplus N^{\prime},
$$

where $N^{\prime}=\sum_{i \geq 0}\left({ }^{t} U_{5}^{0}\right)^{i} h$ with $h=h_{1}$ or $h_{2}$ and ${ }^{t} U_{5}^{0}=x_{2} 1_{p}-{ }^{t} U_{5}^{\prime}$, hence

$$
\operatorname{Coker}\left(\left({ }^{t} \lambda_{3}\right)(p+1, \ldots, b)\right) \cong k(2)^{p} / N^{\prime} .
$$

This $k(2)$-module, however, has infinite length, because it follows from the equation $\eta\left({ }^{t}{ }^{0} U_{5}^{\prime}\right)=0$ for the monic polynomial $\eta(z)=\operatorname{det}$ $\left(z 1_{p}-{ }^{t} U_{5}^{\prime}\right)$ of degree $p$ in a variable $z$ that $N^{\prime}$ is generated over $k(2)$ at most by $p$ elements. This contradicts the condition length ${ }_{R}($ Coker $\left.\left({ }^{t} \lambda_{3}\right)\right)<\infty$ and our assertion is proved.

Q.E.D.

Take a polynomial $f \in I_{a}$, then $n_{1}=\min \left\{\nu \mid(I / f R)_{\nu} \neq\{0\}\right\}$ and there exists a polynomial $g \in I_{n_{1}}$ which is not contained in $f R$. Put $n=n_{1}$. Since $X$ is integral, $f$ and $g$ are relatively prime, so one can use the 
results of $[1 ; \S 3]$ with $J=(f, g) R$ to obtain the generators $g_{0}, g_{1}, \ldots$, $g_{a}, g_{a+1}, \ldots, g_{a+b}$ of $I$ allowing the expression as in [1; Proposition 3. 1], where we may assume $a \leqq n=\operatorname{deg} g_{1} \leqq \ldots \leqq \operatorname{deg} g_{a}, \operatorname{deg} g_{a+1} \leqq \ldots \leqq \operatorname{deg} g_{a+b^{\prime}}$. By abuse of notation we write here $\lambda_{2}=\left[\begin{array}{ccc}U_{01} & U_{02} & 0 \\ U_{1} & U_{2} & U_{4} \\ 0 & U_{3} & U_{5}\end{array}\right]$ to denote the matrix of relations among these generators. In this case $\left[\begin{array}{l}U_{01} \\ U_{1}\end{array}\right]$ is the matrix of relations among the generators $g_{0}, g_{1}, \ldots, g_{a}$ of the ideal $(f, g) R$, so that $\operatorname{rank}_{k}\left[\begin{array}{l}U_{01} \\ U_{1}\end{array}\right](\bmod \mathfrak{m})=a-1$ and one sees deg $g_{i}=n+i-1$ for $1 \leqq i \leqq a$.

Lemma 1.5. In this situation, put $\left(\nu_{a+1}, \ldots, \nu_{a+b^{\prime}}\right)=\left(\operatorname{deg} g_{a+1}, \ldots\right.$, $\left.\operatorname{deg} g_{a+b^{\prime}}\right)$ and $j_{0}=\max \left\{j \mid \nu_{a+j}<n+a-1\right\}$. Then $\nu_{a+1}, \ldots, \nu_{a+j_{0}}$ is a connected sequence of integers and

$$
\operatorname{rank}_{k}\left[{ }^{t} U_{3}{ }^{t} U_{5},{ }^{t} h_{a}^{\prime}\right]\left(j_{0}+1, \ldots, b^{\prime}\right)(\bmod \mathfrak{m}) \geqq q,
$$

where $h_{a}^{\prime}$ is the last row of $U_{4}$ and $q=\#\left\{\nu_{a+1}, \ldots, \nu_{a+j_{0}}\right\}$.

Proof. If the assertion were not true, then, by taking the degrees of the entries into account, one would find for some $p\left(1 \leqq p \leqq j_{0}\right)$ that $U_{3}=\left[\begin{array}{l}U_{3}^{\prime} \\ 0\end{array} *\right], \quad U_{5}=\left[\begin{array}{l}U_{5}^{\prime} \\ 0\end{array} *\right]$ and $h_{a}^{\prime}=(\overbrace{0, \ldots, 0}^{p}, *)$ with $p \times p$ matrices $U_{3}^{\prime}, U_{5}^{\prime}$ such that the entries of $U_{3}^{\prime}-x_{1} 1_{p}$ and $U_{5}^{\prime}-x_{2} 1_{p}$ are in $k(2)$. The situation is almost the same as in the proof of the previous lemma and the argument used there can be applied to this case without any change, consequently $U_{4}\left(p+1, \ldots, b^{\prime}\right)=0$. This implies

$$
\operatorname{Coker}\left(\left({ }^{t} \lambda_{3}\right)\left(p+1, \ldots, b^{\prime}\right)\right) \cong k(2)^{p}
$$

in contradiction with the condition length $\ln _{R}\left(\operatorname{Coker}\left({ }^{t} \lambda_{3}\right)\right)<\infty$, and our assertion is proved.

Q. E. D.

Lemma 1.6. With the notation above, let $d$ and $p_{a}$ be the degree and the arithmetic genus of $X$ respectively. Then

$$
\text { (1.6. 1) } \quad b^{\prime}=a n-d, \sum_{j=1}^{b^{\prime}} \nu_{a+j}=1+\frac{1}{2} a n(a+n-2)-d-p_{a} \text {. }
$$


Proof. Put $\left(\nu_{1}, \ldots, \nu_{a}\right)=\left(\operatorname{deg} g_{1}, \ldots, \operatorname{deg} g_{a}\right)=(n, n+1, \ldots, n+a-1)$. Then the formula of $\left[2 ;\right.$ Remark 1.9] holds true for $\left(a ; \nu_{1}, \ldots, \nu_{a}\right.$; $\left.\nu_{a+1}, \ldots, \nu_{a+b}\right)$ in the notation here, since it is derived only from the direct sum decomposition stated in [2; Proposition 1.3]. The formula (1.6.1) is a consequence of straightforward computations.

Q. E. D.

Example 1.7. (cf. $[4 ; \S 4]$ ). Let $X$ be a nonsingular irreducible curve belonging to the linear system $\left|6 l-\sum_{i=1}^{5} 2 e_{i}\right|$ on a smooth cubic surface in $\mathbf{P}^{3}$, where as usual $l, e_{1}, \ldots, e_{6}$ denote the $Z$-basis of the Picard group of the cubic surface such that $l^{2}=1, e_{i}^{2}=-1$ and $l e_{i}=0$ for $1 \leqq i \leqq 6$. $X$ is a canonical curve with $(d, g)=(8,5)$, and $h^{0}\left(\mathscr{I}_{X}(2)\right)$ $=0, h^{0}\left(\mathscr{I}_{X}(3)\right)=1$ and

$$
h^{0}\left(\mathscr{I}_{X}(4)\right)=\left(\begin{array}{c}
4+3 \\
3
\end{array}\right)-(1-5+32)+h^{1}\left(\mathscr{I}_{X}(4)\right) \geqq 7 .
$$

It follows that $a=3, n=4, b^{\prime}=4$ and $\sum_{j=1}^{4} \nu_{3+j}=18$, by which one finds $\left(a ; \nu_{1}, \ldots, \nu_{a} ; \nu_{a+1}, \ldots, \nu_{a+b^{\prime}}\right)=(3 ; 4,5,6 ; 4,4,5,5)$ combined with Lemma 1.5. This, then, implies

$$
\begin{aligned}
& h^{1}\left(\mathscr{I}_{X}(1)\right)=1, h^{1}\left(\mathscr{I}_{X}(2)\right)=2, h^{1}\left(\mathscr{I}_{X}(3)\right)=1 \\
& \quad \text { and } h^{1}\left(\mathscr{I}_{X}(\nu)\right)=0 \text { for } \nu \geqq 4,
\end{aligned}
$$

so that all elements of $\mathrm{H}_{*}^{1}\left(\mathscr{I}_{X}\right)$ are annihilated at least by two linearly independent linear forms of $R$. The minimal generators of $\mathrm{H}_{*}^{1}\left(\mathscr{I}_{X}\right)$ over $R$ are therefore those of $\mathrm{H}_{*}^{1}\left(\mathscr{I}_{X}\right)$ over $k(2)$ as well, so one sees again by Lemma 1.5 that $B(X)=(3 ; 4,4,4 ; 5)$ or $(3 ; 4,4,5$; $4,5)$ (cf. [2; Proposition 2.4]). But the latter is impossible by Lemma 1.4 , hence $B(X)=(3 ; 4,4,4 ; 5)$. Let $\lambda_{2}$ be the matrix of relations among the generators of $I_{X}$ as in Corollary 1.3, and put ${ }^{t} U_{4}=\left(h_{1}, h_{2}, h_{3}\right)$ with $h_{i} \in k(2)$. Then we see $\left.\operatorname{Hom}_{k}\left(\mathrm{H}_{*}^{1}\left(\mathscr{I}_{X}\right)\right), k\right)$ $\cong k(2)[3] /\left(h_{1}, h_{2}, h_{3}\right) k(2)([2 ;(2.3 .7)])$, and this means by $(1.7 .1)$ that the ideal $\left(h_{1}, h_{2}, h_{3}\right) k(2)$ coincides with $(A, B) k(2)$, where $A, B$ are relatively prime homogeneous polynomials of degree 2 of $k(2)$. We have thus $\mathrm{H}_{*}^{1}\left(\mathscr{I}_{X}\right) \cong k(2)[-1] /(A, B) k(2)$. This curve will be used in the next section to construct the examples we are interested in. 


\section{§2. Nonsingular Irreducible Curves with Basic Sequence $\left(a ; a+2,(a+3)^{a-1} ; a+4\right)$ or $\left(a ;(a+2)^{a-3},(a+3)^{3} ; a+4\right)$ Such That $\mathbf{H}_{*}^{1}(\mathscr{I}) \cong R[-a] /\left(x_{1}, x_{2}, A, B\right) R\left(A, B \in \mathbb{k}(2)_{2}\right)$}

We will make free use of the technique of liaison developped in [8] and [9]. Let's begin with the following lemma.

Lemma 2.1. Let $S$ be a noetherian affine scheme, $p: \mathscr{X}\left(\subset \mathbb{P}_{S}^{3}\right) \longrightarrow S$ a flat family of curves over $S$ and $\mathscr{I}_{\mathscr{X}}$ the ideal sheaf of $\mathscr{X}$ on $\mathbb{P}_{S}^{3}$. Suppose two homogeneous polynomials $\tilde{f}, \tilde{g}$ of $\oplus \mathrm{H}^{0}\left(\mathbb{P}_{S}^{3}, \mathscr{I}_{\mathscr{X}}(\nu)\right) \subset k[S]$ $\left[x_{1}, x_{2}, x_{3}, x_{4}\right]$ define the complete intersection Proj $k^{\nu}(s)\left[x_{1}, x_{2}, x_{3}, x_{4}\right] /\left(\tilde{f}_{s}, \hat{g}_{s}\right)$ for all points $s \in S$, where $k(s)$ is the residue field of the local ring $\left(\mathcal{O}_{s, S}\right.$, $\left.\mathfrak{H t}_{s, s}\right)$ and $\tilde{f}_{s}:=\tilde{f}\left(\bmod \mathfrak{m}_{s, s}\right), \tilde{g}_{s}:=\tilde{g}\left(\bmod \mathfrak{m}_{s, s}\right)$. Then there exists another flat family of curves $q:$ OY $\left(\subset \mathbf{P}_{S}^{3}\right) \longrightarrow S$ such that $\mathscr{Y}_{s}=q^{-1}(s)$ is the curve linked to $\mathscr{X}_{s}=p^{-1}(s)$ by the two surfaces defined by $\tilde{f}_{s}=0$ and $\tilde{g}_{s}=0$ respectively.

Proof. Put $c_{1}=\operatorname{deg} \tilde{f}, c_{2}=\operatorname{deg} \tilde{g}$. Since $\mathcal{O}_{\mathscr{X}}$ is flat over $\mathcal{O}_{S}$, the $\mathcal{O}_{\mathbf{P}_{S}^{3}}$-module $\mathcal{O}_{\mathscr{X}}$ has a locally free resolution

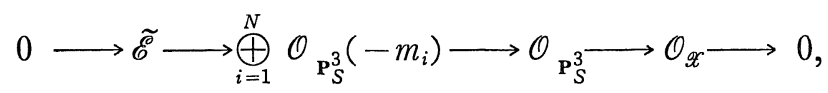

$\widetilde{\mathscr{E}}$ being a vector bundle of $\operatorname{rank} N-1$ on $\mathbb{P}_{S}^{3}$. The sequence

$$
\begin{aligned}
0 \longrightarrow & \mathcal{O}_{\mathbf{P}_{S}^{3}}\left(-c_{1}-c_{2}\right) \stackrel{\left[\begin{array}{r}
g \\
-f
\end{array}\right]}{\longrightarrow} \mathcal{O}_{\mathbf{P}_{S}^{3}}\left(-c_{1}\right) \oplus \mathcal{O}_{\mathbf{P}_{S}^{3}}\left(-c_{2}\right) \\
& \stackrel{(f, g)}{\longrightarrow} \mathcal{O}_{\mathbf{P}_{S}^{3}} \longrightarrow \mathcal{O}_{\mathbf{P}_{S}^{3}} /(\tilde{f}, \tilde{g}) \longrightarrow 0
\end{aligned}
$$

is exact by hypotheses and one can take the mapping cone of the dual of a morphism of complexes from (2.1.2) to (2.1.1), obtaining the complex

$$
\begin{aligned}
0 & \longrightarrow \bigoplus_{i=1}^{N} \mathcal{O}_{\mathbf{P}_{S}^{3}}\left(m_{i}-c_{1}-c_{2}\right) \\
& \longrightarrow \widetilde{\mathscr{E}^{\vee}}\left(-c_{1}-c_{2}\right) \oplus \mathcal{O}_{\mathbf{P}_{S}^{3}}\left(-c_{2}\right) \oplus \mathcal{O}_{\mathbf{P}_{S}^{3}}\left(-c_{1}\right) \stackrel{\psi}{\longrightarrow} \mathcal{O}_{\mathbf{P}_{S}^{3 \cdot}}
\end{aligned}
$$

Since $\tilde{f}, \tilde{g}$ is a regular sequence at each point of $\mathbb{P}_{S}^{3}$, this complex 
proves to be exact (see the proof of [8; Propositions 2.5 and 2.6]). Let $\tilde{\mathscr{I}}$ denote the image of $\phi, \mathscr{Y} \subset \mathbf{P}_{S}^{3}$ the subscheme defined by $\tilde{\mathscr{I}}$ and $q: \mathscr{Y} \longrightarrow S$ the projection. This is the desired family. In fact the ideal sheaf of the curve $Y_{s}^{\prime}$ linked to $\mathscr{X}_{s}$ by the two surfaces $\tilde{f}_{s}=0$ and $\tilde{g}_{s}=0$ coincides with $\operatorname{Im}\left(\psi\left(\bmod \mathfrak{m}_{s, s}\right)\right) \subset \mathcal{O}_{\mathbf{P}_{k(s)}^{3}}$ for each $s \in S$ and the complex (2.1.3) $\left(\bmod \mathfrak{m}_{s, S}\right)$ is exact (loc. cit.), therefore the structure sheaf $\mathcal{O}_{\mathbf{P}_{S}^{3}} / \tilde{\mathscr{I}}$ of $\mathscr{Y}$ is flat over $\mathcal{O}_{s}$ and $Y_{s}^{\prime}=\mathscr{Y}_{s}$, which proves our assertion.

Q.E.D.

From now on $M$ will denote the $R$-module $R /\left(x_{1}, x_{2}, A, B\right) R$ defined by relatively prime homogeneous polynomials $A, B \in k(2)$ of degree 2 .

Lemma 2.2. Let $a, n_{1}, \ldots, n_{a}, n_{a+1}$ be integers satisfying the condition $a \leqq n_{1} \leqq \ldots \leqq n_{a-2}=n_{a-1}=n_{a}, n_{a+1}=n_{a}+1$. Then the curves $X$ with $B(X)$ $=\left(a ; n_{1}, \ldots, n_{a} ; n_{a+1}\right)$ such that $\mathrm{H}_{*}^{1}\left(\mathscr{I}_{X}\right) \cong M\left[-\left(n_{a+1}-4\right)\right]$ form an irreducible subset of $\operatorname{Hilb}\left(\mathbf{P}^{3}\right)$ and the ideal $I_{X}$ is generated over $R$ by elements of degrees at most $n_{a}$ for every $X$ corresponding to a general point of this subset.

Proof. Let $f_{0}, f_{1}, \ldots, f_{a}, f_{a+1}$ be the generators of $I_{X}$ that give the sequence $B(X)$ and let $\lambda_{2}, \lambda_{3}$ be the matrices as described in [2; Proposition 1.3]. The condition $\mathrm{H}_{*}^{1}\left(\mathscr{I}_{X}\right) \cong M\left[-\left(n_{a+1}-4\right)\right]$ implies $\operatorname{Im}^{k(2)}\left({ }^{t} U_{4}\right)=(A, B) R$, so that we may assume ${ }^{t} U_{4}=(0, \ldots, 0, A, B)$ by changing $\left(f_{1}, \ldots, f_{a}\right)$ for $\left(f_{1}^{\prime}, \ldots, f_{a}^{\prime}\right):=\left(f_{1}, \ldots, f_{a}\right) G$ with a suitable $G \in G L(a, k(2))$. Since the relations among $A, B$ are generated $\mathrm{b}:=$ ${ }^{t}(-B, A)$, the space $T$ which parameterizes $\lambda_{2}$ satisfying the equation $\lambda_{2} \lambda_{3}=0$ is irreducible (cf. [1; Remark 4.1]), and $U_{21}$ takes the form $(*, c, 0,0)$ for $\lambda_{2}$ corresponding to a general point of $T$, where $c$ is a nonzero element of $k$. Let $\Phi: T \longrightarrow \operatorname{Hilb}\left(\mathbf{P}^{3}\right)$ be the natural morphism which induces the family of curves over $T$ defined by the ideals arising from these $\lambda_{2}$. Then it is enough to regard $\Phi(T)$ as the irreducible subset in the statement.

Q.E.D.

Proposition 2.3. Let $a$ be an integer larger than or equal to 4, and suppose a nonsingular irreducible curve $X$ satisfying $B(X)=\left(a-1 ; a^{a-1}\right.$; $a+1), \mathrm{H}_{*}^{1}\left(\mathscr{I}_{X}\right) \cong M[-(a-3)]$ exists. We assume furthermore that $I_{X}$ is generated over $R$ by elements of degrees at most a. 
1) Let $Y_{1}$ be the curve linked to $X$ by the two surfaces defined respectively by a general element of $I_{X, a}$ and of $I_{X, a+1}$. Then $Y_{1}$ is nonsingular irreducible with $B\left(Y_{1}\right)=\left(a ; a^{a-3},(a+1)^{3} ; a+2\right), \mathrm{H}_{*}^{1}\left(\mathscr{I}_{Y_{1}}\right) \cong M[-(a-2)]$.

2) Let $Z_{1}$ be the curve linked to $Y_{1}$ by the two surfaces both defined by a general element of $I_{Y_{1}, a+1}$. Then $Z_{1}$ is nonsingular irreducible with $B\left(Z_{1}\right)=\left(a ;(a+1)^{a} ; a+2\right), \mathrm{H}_{*}^{1}\left(\mathscr{I}_{Z_{1}}\right) \cong M[-(a-2)]$.

3) For an integer $n(n \geqq a+2)$, let $Y_{2}$ be the curve linked to $X$ by the two surfaces defined respectively by a general element of $I_{X, a}$ and of $I_{X, n}$. Then $Y_{2}$ is nonsingular irreducible with $B\left(Y_{2}\right)=\left(a ;(n-1)^{a-3}, n^{3} ; n+1\right)$, $\mathrm{H}_{*}^{1}\left(\mathscr{I}_{Y_{2}}\right) \cong M[-(n-3)]$.

4) Let $Z_{2}$ be the curve linked to $Y_{2}$ by the two surfaces defined respectively by a general element of $I_{Y_{2}, a}$ and of $I_{Y_{2}, 2 n-a}$. Then $Z_{2}$ is nonsingular irreducible with $B\left(Z_{2}\right)=\left(a ; n-1, n^{a-1} ; n+1\right), \mathrm{H}_{*}^{1}\left(\mathscr{I}_{Z_{2}}\right) \cong M[-(n-3)]$. Furthermore, in all these four cases we may assume that the ideals $I_{Y_{1}}$ and $I_{Z_{1}}$ (resp. $I_{Y_{2}}$ and $I_{Z_{2}}$ ) are generated over $R$ by elements of degrees at most $a+1$ (resp. n).

Proof. Since the argument is common to all cases, we only give the proof of 4) assuming the results of 3). Notice first that a general element of $I_{X, a}$ defines a smooth surface. It then follows from the definition of $Y_{2}$ that a general element of $I_{Y_{2}, a}$ also defines a smooth surface, and since $I_{Y_{2}}$ is generated over $R$ by elements of degrees at most $n$, the curve $Z_{2}$ is nonsingular (see the proof of [8; Proposition 4. 1] under the condition char. $k=0)$. In addition, one has $\mathrm{H}_{*}^{1}\left(\mathscr{I}_{Z_{2}}\right)$ $\cong \operatorname{Ext}_{R}^{4}\left(H_{*}^{1}\left(\mathscr{I}_{Y_{2}}\right), R\right)[-2 n] \cong M[-(n-3)]$, so $h^{1}\left(\mathscr{I}_{Z_{2}}\right)=0$, whence $Z_{2}$ is nonsingular irreducible.

Let

$$
0 \longrightarrow \mathscr{E} \longrightarrow \mathcal{O}_{\mathbf{P}^{3}}\left(-a,(-n+1)^{a-3},(-n)^{3},-n-1\right) \longrightarrow \mathscr{I}_{Y_{2}} \longrightarrow 0
$$

be the locally free resolution of $Y_{2}$, where we have set

$$
\mathcal{O}_{\mathbf{P}^{3}}\left(m_{1}, \ldots, m_{s}\right)=\bigoplus_{i=1}^{s} \mathcal{O}_{\mathbf{P}^{3}}\left(m_{i}\right)
$$

for simplicity. Then $\mathscr{I}_{Z_{2}}$ has a locally free resolution of the form

$$
0 \longrightarrow \mathcal{O}_{\mathbf{P}^{3}}\left(a-2 n,(-n-1)^{a-3},(-n)^{3},-n+1\right)
$$




$$
\longrightarrow \mathcal{O}_{\mathbf{P}^{3}}(a-2 n,-a) \oplus \mathscr{E}^{\vee}(-2 n) \longrightarrow \mathscr{I}_{Z_{2}} \longrightarrow 0 .
$$

Notice the free resolution of $I_{Y_{2}}$ yields the exact sequence

$$
\begin{aligned}
& 0 \longrightarrow \mathscr{E}^{\vee}(-2 n) \longrightarrow \mathcal{O}_{\mathbf{P}^{3}}\left((-n)^{a-3},(-n+1)^{3},(-n+2)^{2}\right) \\
& \stackrel{{ }^{t} \lambda_{3}}{\longrightarrow} \mathcal{O}_{\mathbf{P}^{3}}(-n+3) \longrightarrow 0
\end{aligned}
$$

with ${ }^{t} \lambda_{3}=\left(0, \ldots, 0, A, B,-x_{2}, x_{1}\right)$. Since $\mathrm{H}_{*}^{1}\left(\mathscr{I}_{Z_{2}}\right) \cong M[-(n-3)]$, one sees $\bar{n}^{2}$ of $B\left(Z_{2}\right)$ is $n+1$ (cf. [2; Proposition 2.4]). On the other hand, it follows from the sequences (2.3.1) and (2.3.2) that $h^{0}\left(\mathscr{I}_{Z_{2}}\right.$ $(\nu))=0$ for $\nu \leqq a-1, h^{0}\left(\mathscr{I}_{z_{2}}(\nu)\right)=\left(\begin{array}{c}\nu-a+3 \\ 3\end{array}\right)$ for $a \leqq \nu \leqq n-2, h^{0}\left(\mathscr{I}_{z_{2}}(n\right.$ $-1))=\left(\begin{array}{c}n-a+2 \\ 3\end{array}\right)+1$ and $h^{0}\left(\mathscr{I}_{Z_{2}}(n)\right)=\left(\begin{array}{c}n-a+3 \\ 3\end{array}\right)+a+2$, consequently $B\left(Z_{2}\right)=\left(a ; n-1, n^{a-1} ; n+1\right)$.

It remains to show that we may assume $I_{Z_{2}}$ is generated over $R$ by elements of degrees at most $n$. For this purpose we use Lemma 2.2 to construct the family $p: \mathscr{X} \longrightarrow S$ over an integral affine scheme $S$ arising from the flat deformation of the ideal $I_{Z_{2}}$, such that $I_{\mathscr{X}_{s}}$ is generated over $R$ by elements of degrees at most $n$ for every general point $s \in S$ and $\mathscr{X}_{s_{1}}=Z_{2}$ for a point $s_{1} \in S$. Next take two homogeneous polynomials $\tilde{f} \in I_{\mathscr{X}, a}, \tilde{g} \in I_{\mathscr{O}, 2 n-a}$ such that the curve linked to $Z_{2}$ by the surfaces defined respectively by $\tilde{f}_{s_{1}}=0$ and $\tilde{g}_{s_{1}}=0$ coincides with $Y_{2}$. Using these polynomials, construct then a flat family $q: \mathscr{Y} \longrightarrow S$ which has the properties stated in Lemma 2.1. The general fibers $\mathscr{Y}_{s}$ are nonsingular irreducible, and since $\mathrm{H}_{*}^{1}\left(\mathscr{I}_{\mathscr{O}_{s}}\right) \cong \mathrm{H}_{*}^{1}\left(\mathscr{I}_{Y_{1}}\right)$ for all points $s \in S$, we have $B\left(\mathscr{Y}_{s}\right)=B\left(Y_{1}\right)$, therefore we may replace $Y_{2}$ with $\mathscr{Y}_{s_{0}}$ and $Z_{2}$ with $\mathscr{X}_{s_{0}}$ for a suitable point $s_{0} \in S$, from which our assertion follows.

Q.E.D.

In the same manner one obtains the following.

Proposition 2.4. Let $a$ and $b$ be integers such that $a \geqq 2 b, b \geqq 1$. Suppose a nonsingular a. B. curve (see $[2 ; \S 3]) X$ with $B(X)=\left(a ; a^{a} ; a^{b}\right)$ exists.

1) Let $Y_{1}$ be the curve linked to $X$ by the two surfaces defined respectively by a general element of $I_{X, a}$ and of $I_{X, a+1}$. Then $Y_{1}$ is a nonsingular irreducible 
a. $B$. curve with $B\left(Y_{1}\right)=\left(a ; a^{a-2 b},(a+1)^{2 b} ;(a+1)^{b}\right)$.

2) Let $Z_{1}$ be the curve linked to $Y_{1}$ by the two surfaces both defined by a general element of $I_{Y_{1}, a+1}$. Then $Z_{1}$ is a nonsingular irreducible $a_{0} B$. curve with $B\left(Z_{1}\right)=\left(a+1 ;(a+1)^{a+1} ;(a+1)^{b}\right)$.

3) Let $n$ be an integer bigger than $a$, and let $Y_{2}$ be the curve linked to $X$ by the two surfaces defined respectively by a general element of $I_{X, a}$ and of $I_{X, n}$. Then $Y_{2}$ is a nonsingular irreducible $a$. B. curve with $B\left(Y_{2}\right)=(a$; $\left.(n-1)^{a-2 b}, n^{2 b} ; n^{b}\right)$.

4) Let $Z_{2}$ be the curve linked to $Y_{2}$ by the two surfaces defined respectively by a general element of $I_{Y_{2^{\prime}} a}$ and of $I_{Y_{2}, 2 n-a}$. Then $Z_{2}$ is a nonsingular irreducible a. $B$. curve with $B\left(Z_{2}\right)=\left(a ; n^{a} ; n^{b}\right)$.

Remark 2.5. The basic sequences of $Z_{1}$ and $Z_{2}$ appeared in the propositions above may be computed generally as follows. Let $X$ be a curve in $\mathbb{P}^{3}$ with $B(X)=\left(a ; \bar{n}^{1} ; \bar{n}^{2}\right), Y$ the curve obtained by applying linkage to $X$ with two surfaces of degrees $s_{1}, s_{2}$ respectively and $Z$ the curve obtained by applying linkage to $Y$ with two surfaces of degrees $t_{1}, t_{2}$ respectively. Then we deduce from the locally free resolution for $\mathscr{I}_{X}$

$$
0 \longrightarrow \mathscr{E} \longrightarrow \mathcal{O}_{\mathbf{P}^{3}}\left(-a,-\bar{n}^{1},-\bar{n}^{2}\right) \longrightarrow \mathscr{I}_{X} \longrightarrow 0
$$

the locally free resolution for $\mathscr{I}_{Z}$ of the following form

$$
\begin{aligned}
0 \longrightarrow & \mathscr{E}(u) \oplus \mathcal{O}_{\mathbf{P}^{3}}\left(s_{2}-t, s_{1}-t\right) \\
& \longrightarrow \mathcal{O}_{\mathbf{P}^{3}}\left(u-a, u-\bar{n}^{1}, u-\bar{n}^{2}\right) \oplus \mathcal{O}_{\mathbf{P}^{3}}\left(-t_{2},-t_{1}\right) \longrightarrow \mathscr{I}_{2} \longrightarrow 0
\end{aligned}
$$

(see the proof of $[9 ;(1.7)$ Theorem $]$ ), and we have $\mathrm{H}_{*}^{1}\left(\mathscr{I}_{Z}\right) \cong \operatorname{Ext}_{R}^{4}$ $\left(\operatorname{Ext}_{R}^{4}\left(\mathrm{H}_{*}^{1}\left(\mathscr{I}_{X}\right), R\right),[-s], R\right)[-t] \cong \mathrm{H}_{*}^{1}\left(\mathscr{I}_{X}\right)[u]$, where $s=s_{1}+s_{2}, \quad t=t_{1}$ $+t_{2}$ and $u=s-t$. Suppose $s_{1}=a, s_{2}=t_{1}=t_{2}=a+1$. Then the resolution becomes

$$
\begin{aligned}
0 \longrightarrow \mathscr{E}(-1) \oplus \mathcal{O}_{\mathbf{p}^{3}}(-a-1,-a-2) \\
\longrightarrow \mathcal{O}_{\mathbf{P}^{3}}\left(-a-1,-\bar{n}^{1}-1,-\bar{n}^{2}-1\right) \oplus \mathcal{O}_{\mathbf{P}^{3}}(-a-1,-a-1) \rightarrow \mathscr{I}_{2} \rightarrow 0
\end{aligned}
$$

and $\mathrm{H}_{*}^{1}\left(\mathscr{I}_{Z}\right) \cong H_{*}^{1}\left(\mathscr{J}_{X}\right)[-1]$, from which follows $B(Z)=(a+1 ; a+1$, $\left.\bar{n}^{1}+1 ; \bar{n}^{2}+1\right)$. Suppose next $s_{1}=t_{1}=a, s_{2}=n, t_{2}=2 n-a(n>a)$. Then the resolution becomes

$$
\begin{aligned}
0 \longrightarrow & \mathscr{E}(a-n) \oplus \mathcal{O}_{\mathbf{P}^{3}}(-n, a-2 n) \\
& \longrightarrow \mathcal{O}_{\mathbf{P}^{3}}\left(-n, a-n-\bar{n}^{1}, a-n-\bar{n}^{2}\right) \oplus \mathcal{O}_{\mathbf{P}^{3}}(a-2 n,-a) \longrightarrow \mathscr{I}_{Z} \longrightarrow 0
\end{aligned}
$$


and $\mathrm{H}_{*}^{1}\left(\mathscr{I}_{Z}\right) \cong \mathrm{H}_{*}^{1}\left(\mathscr{I}_{X}\right)[-(n-a)]$, from which follows $B(Z)=(a$; $\left.\bar{n}^{1}+n-a ; \bar{n}^{2}+n-a\right)$.

Corollary 2.6. (cf. [2; Theorem 4.4]). The following basic sequences are realized by nonsingular irreducible a. B. curves: $\left(a ; n^{a} ; n^{b}\right)$ and $(a$; $\left.(n-1)^{a-2 b}, n^{2 b} ; n^{b}\right)$ for $a, b, n$ satisfying $n>a \geqq 2 b, b \geqq 1,\left(a ; a^{a} ; a^{b}\right)$ for $a, b$ satisfying $a \geqq 2 b, b \geqq 2$ or $a \geqq 3, b=1$.

Proof. In view of Proposition 2. 4 it suffices to show the existence of nonsingular a. B. curves with basic sequence $\left(2 ; 2^{2} ; 2\right)$ and the existence of nonsingular irreducible a. B. curves with basic sequence $\left(2 b ;(2 b)^{2 b} ;(2 b)^{b}\right)$ for $b \geqq 2$. The proof can be found in [3; Section 5], but we write it here for the sake of completeness. First define the vector bundle $\mathscr{F}$ of rank $3 b+1$ as the cokernel of the map

$$
v: \mathcal{O}_{\mathbf{P}^{3}}(-1)^{\oplus b} \longrightarrow \mathcal{O}_{\mathbf{P}^{3}}(1) \oplus \mathcal{O}_{\mathbf{P}^{3}}^{\oplus 4 b}
$$

where $b \geqq 1$ and ${ }^{t} v=\left(0, x_{1} 1_{b}, x_{2} 1_{b}, x_{3} 1_{b}, x_{4} 1_{b}\right)$. Then $\mathscr{F}^{\vee}(1)$ is generated by its global sections and general $3 b$ linearly independent elements of $\mathrm{H}^{0}\left(\mathscr{F}^{\vee}(1)\right)$ define an ideal sheaf $\mathscr{I}_{X}$ of a curve $X$ in $\mathbf{P}^{3}$ that fits in with the exact sequence

$$
0 \longrightarrow \mathcal{O}_{\mathbf{P}^{3}}(-2 b)^{\oplus 3 b} \longrightarrow \mathscr{F}^{\vee}(1-2 b) \cong \bigwedge^{3 b} \mathscr{F} \otimes \mathcal{O}_{\mathbf{P}^{3}}(-3 b) \longrightarrow \mathscr{I}_{X} \longrightarrow 0
$$

by the standard method (cf. $[2 ; \S 2]$ ). Since $h^{0}\left(\mathscr{I}_{X}(\nu)\right)=0$ for $\nu<2 b$, $h^{0}\left(\mathscr{I}_{X}(2 b)\right)=3 b+1$ and $\mathrm{H}_{*}^{1}\left(\mathscr{I}_{X}\right) \cong \mathrm{H}_{*}^{1}\left(\mathscr{F}^{\vee}(1-2 b)\right) \cong k[2-2 b]^{b}$, we find $B(X)=\left(2 b ;(2 b)^{2 b} ;(2 b)^{b}\right)$. This curve is in fact nonsingular if the $3 b$ sections are chosen sufficiently generally, for the Kleiman's version of Bertini's theorem [7; Theorem (3.3)] is valid for the sections of the vector bundle $\mathscr{F}^{\vee}(1)$ itself in characteristic $0 . \quad$ Q. E. D.

Starting from the nonsingular irreducible curve described in Example 1.7, one obtains, by applying Proposition 2.4 successively with $n=a+3$, nonsingular irreducible curves $X, Y_{2}$ and $Z_{2}$ satisfying $B(X)=\left(a-1 ; a^{a-1} ; a+1\right), \mathrm{H}_{*}^{1}\left(\mathscr{I}_{X}\right) \cong M[-(a-3)], B\left(Y_{2}\right)=\left(a ;(a+2)^{a-3}\right.$, $\left.(a+3)^{3} ; a+4\right), B\left(Z_{2}\right)=\left(a ; a+2,(a+3)^{a-1} ; a+4\right)$ and $\mathrm{H}_{*}^{1}\left(\mathscr{I}_{Y_{2}}\right) \cong \mathrm{H}_{*}^{1}\left(\mathscr{I}_{Z_{2}}\right)$ $\cong M[-a]$ for all $a \geqq 4$. It will be shown that the points of $\operatorname{Hilb}\left(\mathbf{P}^{3}\right)$ corresponding to the curves $Y_{2}$ or $Z_{2}$ are contained at least in two irreducible components of $\mathrm{Hilb}\left(\mathbf{P}^{3}\right)$. We first prove that they are 
deformed flatly into a. B. curves.

Proposition 2.7. Let $a$ be an integer larger than or equal to 4. Suppose every nonsingular irreducible curve $X$ with $B(X)=\left(a-1 ; a^{a-1} ; a+1\right), \mathrm{H}_{*}^{1}$ $\left(\mathscr{I}_{X}\right) \cong M[-(a-3)]$ can be deformed flatly into an $a . B$. curve with basic sequence $\left(a ; a^{a} ; a^{2}\right)$. Then we have the following.

1) Every nonsingular irreducible curve $Y_{1}$ with $B\left(Y_{1}\right)=\left(a ; a^{a-3},(a+1)^{3}\right.$; $a+2), \mathrm{H}_{*}^{1}\left(\mathscr{I}_{Y_{2}}\right) \cong M[-(a-2)]$ can be deformed flatly into an $a$. B. curve with basic sequence $\left(a ; a^{a-4},(a+1)^{4} ;(a+1)^{2}\right)$.

2) It follows from 1) that every nonsingular irreducible curve $Z_{1}$ with $B\left(Z_{1}\right)=\left(a ;(a+1)^{a} ; a+2\right), \mathrm{H}_{*}^{1}\left(\mathscr{I}_{Z_{1}}\right) \cong M[-(a-2)]$ can be deformed flatly into an $a . B$. curve with basic sequence $\left(a+1 ;(a+1)^{a+1} ;(a+1)^{2}\right)$.

3) Every nonsingular irreducible curve $Y_{2}$ with $B\left(Y_{2}\right)=\left(a ;(a+2)^{a-3}\right.$, $\left.(a+3)^{3} ; a+4\right), \mathrm{H}_{*}^{1}\left(\mathscr{I}_{Y_{2}}\right) \cong M[-a]$ can be deformed flatly into an $a . B$. curve with basic sequence $\left(a ;(a+2)^{a-4},(a+3)^{4} ;(a+3)^{2}\right)$.

4) It follows from 3) that every nonsingular irreducible curve $Z_{2}$ with $B\left(Z_{2}\right)=\left(a ; a+2,(a+3)^{a-1} ; a+4\right), \mathrm{H}_{*}^{1}\left(\mathscr{I}_{Z_{2}}\right) \cong M[-a]$ can be deformed flatly into an $a$. $B$. curve with basic sequence $\left(a ;(a+3)^{a} ;(a+3)^{2}\right)$.

Proof. Since the argument is common to all cases, we only give the proof for 4) assuming the results of 3). Let $S^{\prime}$ (resp. $S^{\prime \prime}$ ) be the irreducible subspace of $\mathrm{Hilb}\left(\mathbf{P}^{3}\right)$ whose points correspond to nonsingular irreducible curves $Z$ (resp. $Y$ ) with $B(Z)=\left(a ; a+2,(a+3)^{a-1} ; a+4\right)$ (resp. $\left.B(Y)=\left(a ;(a+2)^{a-3},(a+3)^{3} ; a+4\right)\right)$ such that $\mathrm{H}_{*}^{1}\left(\mathscr{I}_{Z}\right) \cong M[-a]$ (resp. $\mathrm{H}_{*}^{1}\left(\mathscr{I}_{Y}\right) \cong M[-a]$ ) (see Lemma 2.2). And let $H^{\prime}$ (resp. $H^{\prime \prime}$ ) be the irreducible component of $\mathrm{Hilb}\left(\mathbb{P}^{3}\right)$ whose general points correspond to a. B. curves with basic sequence $\left(a ;(a+3)^{a} ;(a+3)^{2}\right)$ (resp. $\left(a ;(a+2)^{a-4},(a+3)^{4} ;(a+3)^{2}\right)($ see [2; Theorem 5.11]). Then the results of 3) implies that $S^{\prime \prime}$ is contained in $H^{\prime \prime}$. Now let $Z_{2}$ be a curve corresponding to a general point of $S^{\prime}$. We may assume, arguing as in the last part of the proof of Proposition 2.3, that $Z_{2}$ is linked to a nonsingular irreducible curve $Y_{2}$ which corresponds to a point of $S^{\prime \prime}$ by the two surfaces defined respectively by a general element $f$ of $I_{Y_{2^{\prime}} a}$ and $g$ of $I_{Y_{2}, a+6}$. Let $S$ be a sufficiently small affine subset of $H^{\prime \prime}$ which contains the point $s_{2}$ corresponding to the curve $Y_{2}$, and let $p$; $\mathscr{X} \longrightarrow S$ be the family of curves over $S$ induced by the universal 
family over $\operatorname{Hilb}\left(\mathbf{P}^{3}\right)$. Since $h^{0}\left(\mathscr{I}_{Y_{2}}(a)\right)=h^{0}\left(\mathscr{I}_{\mathscr{X}_{s}}(a)\right)=1$ for all general $s \in S$ and since $h^{1}\left(\mathscr{I}_{Y_{2}}(a+6)\right)=0$, there exist homogeneous polynomials $\tilde{g} \in \mathrm{H}^{0}\left(\mathscr{I}_{\mathscr{C}}(a)\right)$ and $\tilde{g} \in \mathrm{H}^{0}\left(\mathscr{I}_{\mathscr{X}}(a+6)\right)$ such that $\tilde{f}_{s_{2}}=f$ and $\tilde{g}_{s_{2}}=g$, which allows us to construct another flat family $q: \mathscr{Y} \longrightarrow S$ having the properties stated in Lemma 2.1. In this family, $\mathscr{Y}_{s}$ is an a. B. curve with basic sequence $\left(a ;(a+3)^{a} ;(a+3)^{2}\right)$ for every general $s \in S$ by Proposition 2.4 and $\mathscr{Y}_{s_{2}}=Z_{2}$, therefore $Z_{2}$ is deformed into an a. B. curve. Since $Z_{2}$ corresponds to a general point of $S^{\prime}$ by assumption, we have $S^{\prime} \subset H^{\prime}$, which proves the assertion.

Q. E. D.

When the a. B. curves with basic sequence $\left(a ; \bar{n}^{1} ; \bar{n}^{2}\right)$ form a Zariski open set of an irreducible component of $\operatorname{Hilb}\left(\mathbf{P}^{3}\right)$, we will denote this component by $\overline{\mathrm{H}}_{\text {a. в. }}\left(a ; \bar{n}^{1} ; \bar{n}^{2}\right)$.

Corollary 2.8. Let $a$ be an integer larger than or equal to 4. Then the subset of $\mathrm{Hilb}\left(\mathbb{P}^{3}\right)$ corresponding to nonsingular irreducible curves $X$ with basic sequence $\left(a ; a+2,(a+3)^{a-1} ; a+4\right)$ (resp. $\left(a ;(a+2)^{a-3},(a+3)^{3}\right.$; $a+4)$ ) and such that $\mathrm{H}_{*}^{1}\left(\mathscr{I}_{X}\right) \cong M[-a]$ is contained in $\overline{\mathrm{H}}_{a . B .}\left(a ;(a+3)^{a}\right.$; $\left.(a+3)^{2}\right)\left(\operatorname{resp} . \overline{\mathrm{H}}_{a . B .}\left(a ;(a+2)^{a-4},(a+3)^{4} ;(a+3)^{2}\right)\right) . \quad($ See $[2 ;$ Theorem 5. 11].)

Proof. It is enough to show that every nonsingular irreducible curve $C$ with $B(C)=\left(3 ; 4^{3} ; 5\right), \mathrm{H}_{*}^{1}\left(\mathscr{I}_{C}\right) \cong M[-1]$ can be deformed flatly into an a. B. curve with basic sequence $\left(4 ; 4^{4} ; 4^{2}\right)$, because one can prove the assertion with the use of Proposition 2.7 starting from this fact. For this purpose we have only to borrow the results of [4; $\S 4]$. One knows that nonsingular irreducible a. B. curves with basic sequence $\left(4 ; 4^{4} ; 4^{2}\right)$ exist (Corollary 2.6) and that they form a Zariski open set of the irreducible component $\overline{\mathrm{H}}_{a . B .}\left(4 ; 4^{4} ; 4^{2}\right)$. Besides, this irreducible component coincides with the closure of $\mathrm{H}_{8,5}$ (see Introduction) in $\operatorname{Hilb}\left(\mathbb{P}^{3}\right)$ by $[4 ; \S 4]$ (see $[6 ;$ p. 75] also), and contains the point corresponding to $C$. This proves our assertion. Q. E. D.

The following result, combined with the corollary above, gives what we wanted.

Proposition 2.9. Let $X$ be a curve with $B(X)=\left(a ;(a+2)^{a^{\prime}},(a+3)^{a^{\prime \prime}}\right.$; 
$a+4), \mathrm{H}_{*}^{1}\left(\mathscr{I}_{X}\right) \cong M[-a]$, where $a^{\prime}$, $a^{\prime \prime}$ are integers satisfying $a^{\prime}+a^{\prime \prime}$ $=a, a^{\prime} \geqq 1, a^{\prime \prime} \geqq 2$. Then $X$ can be deformed flatly into a projectively Cohen-Macaulay curve with basic sequence $\left(a+1 ; a+1,(a+2)^{a^{\prime}+2},(a+3)^{a^{\prime \prime}-2}\right)$.

Proof. Compute the free resolution for the $R$-module $\mathrm{H}_{*}^{0}\left(\mathcal{O}_{X}\right)$ following the method of $[2 ; \S 2]$. It is of the form

$$
0 \longrightarrow R\left[-\bar{m}_{2}\right] \stackrel{\tau}{\longrightarrow} R\left[-\bar{m}_{1}\right] \stackrel{\sigma}{\longrightarrow} R \oplus R[-a] \stackrel{\rho}{\longrightarrow} \mathrm{H}_{*}^{0}\left(\mathcal{O}_{X}\right) \longrightarrow 0
$$

where

$$
\bar{m}_{1}=\left(a, a+1,(a+2)^{a^{\prime}},(a+3)^{a^{\prime \prime}-2}, a+1,(a+2)^{2}\right)
$$

and

$$
\bar{m}_{2}=\left((a+3)^{a^{\prime}},(a+4)^{a^{\prime \prime}-2}, a+2,(a+3)^{2}\right)
$$

(see Propositions 2.8 and 2.9 of [2]).

Let $f_{0}, f_{1}, \ldots, f_{a}, f_{a+1}$ be the generators of $I_{X}$ associated with $B(X)$ as usual. Then we may assume that $\sigma$ is of the form

$$
\left[\begin{array}{cccccc}
f_{0} & s_{1} & f_{1}, \ldots, f_{a-2} & s_{2} & s_{3} & s_{4} \\
\hdashline 0 & x_{1} & 0 & x_{2} A & B
\end{array}\right]
$$

so

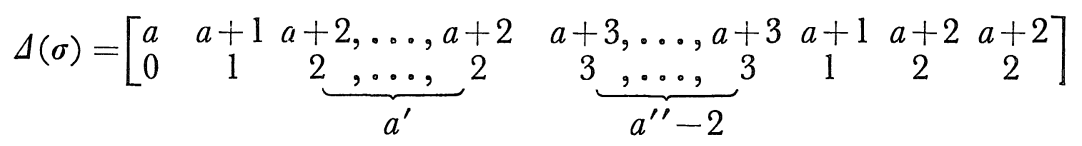

and

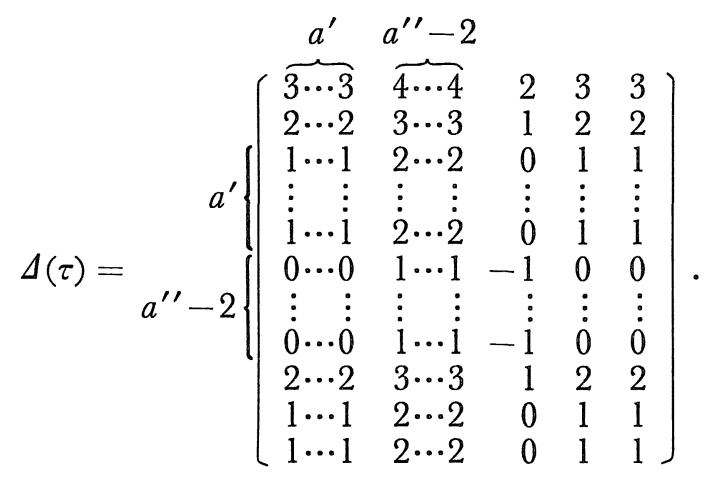

Write the $\left(a^{\prime}+a^{\prime \prime}-1\right)$-th column of $\tau$ as follows: ${ }^{t}\left(x_{1} h_{1}^{(1)}+x_{2} h_{1}^{(2)}+h_{1}^{(3)}\right.$, $\left.h_{2}, h_{3}, \ldots\right)$, where $h_{1}^{(3)} \in k(2)$ and $h_{3} \in k$. Let $t$ be a parameter. Put 


$$
\begin{aligned}
& \tilde{\tilde{\sigma}}=(\left(t+h_{3}\right) t, x_{1},-t h_{1}^{(3)}, \overbrace{0, \ldots, 0}^{a-3}, x_{2}, A, B), \\
& \bar{\sigma}=\sigma(l)\left(0, x_{1}, 0, \ldots, 0, x_{2}, A, B\right)
\end{aligned}
$$

and we define $\tau_{1}$ to be the matrix whose $i$-th column is that of $\tau$ for each $i \neq a^{\prime}+a^{\prime \prime}-1$ and whose $\left(a^{\prime}+a^{\prime \prime}-1\right)-t h$ column is ${ }^{t}\left(x_{1} h_{1}^{(1)}+x_{2} h_{1}^{(2)}\right.$ $\left.+h_{1}^{(3)}, h_{2}, t+h_{3}, \ldots\right)$. In this setting, since $M_{\nu}=0$ for $\nu \geqq 3$, we find there exists a matrix $\tau_{2}$ with entries in $t R[t]$ which satisfies the equation $\tilde{\tilde{\sigma}} \tau_{1}=\bar{\sigma} \tau_{2}$ and such that the degree of its $(i, j)$-component with respect to $x_{1}, x_{2}, x_{3}, x_{4}$ is the same as that of $\tau$. We may assume here that the $i$-th row of $\tau_{2}$ is zero for $i=1$ and $3 \leqq i \leqq a$. Put $\tilde{\tau}=\tau_{1}-\tau_{2}$, then we have $\tilde{\tilde{\sigma}} \tilde{\tau}=0, \tilde{\tau}(\bmod t R[t])=\tau, \quad \tilde{\sigma}(\bmod t R[t])=\bar{\sigma}$ and $\mathscr{Z}:=\operatorname{Proj}_{k[t]}$ $(R[t] / I(\tilde{\tau}))$ gives a flat family of curves $p: \mathscr{Z}\left(\subset \mathbf{P}_{k[t]}^{3}\right) \longrightarrow \operatorname{Spec} k[t]$ such that $\mathscr{Z}_{\mathcal{O}}=X$ by [2; Proposition 2.11], where $I(\tilde{\tau})$ is the ideal in $R[t]$ generated by the maximal minors of $\tilde{\tau}$, and $o$ denotes the point defined by $t=0$. It follows from the isomorphism $\mathrm{H}_{*}^{1}\left(\mathscr{I}_{\mathscr{Z}_{s}}\right) \cong$ $R[-a] / \operatorname{Im}^{R}(\tilde{\tilde{\sigma}})$ (loc. cit.) that $\mathrm{H}_{*}^{1}\left(\mathscr{I}_{\mathscr{Z}_{s}}\right)$ becomes zero for general points $s \in$ Spec $k[t]$, which implies that $X$ can be deformed flatly into a projectively Cohen-Macaulay curve. In order to find the basic sequence of $\mathscr{Z}_{s}$ for a general point $s$, it is enough to notice that $I_{\mathscr{L}_{s}}$ has a free resolution of the form

$$
\begin{gathered}
0 \longrightarrow R\left[-\bar{m}_{2}\right] \stackrel{\left.\tilde{\tau}{ }^{1}\right)}{\longrightarrow} R\left[-a-1,(-a-2)^{a^{\prime}},(-a-3)^{a^{\prime \prime}-2},\right. \\
\left.-a-1,(-a-2)^{2}\right] \longrightarrow I_{\mathscr{Z}_{a}} \longrightarrow 0 .
\end{gathered}
$$

Q. E. D.

Let $\overline{\mathrm{H}}\left(a ; n_{1}, \ldots, n_{a}\right)$ denote the irreducible component of $\mathrm{Hilb}\left(\mathbf{P}^{3}\right)$ whose general points correspond to projectively Cohen-Macaulay curves with basic sequence $\left(a ; n_{1}, \ldots, n_{a}\right)$ where $a \leqq n_{1} \leqq \ldots \leqq n_{a}$ (cf. [4; Proposition 2.10]). All that we have done can be summarized as follows.

Proposition 2.10. Let $a$ be an integer larger than or equal to 4 .

1) $\overline{\mathrm{H}}\left(a+1 ; a+1,(a+2)^{a-1}, a+3\right) \cap \overline{\mathrm{H}}_{\text {а. в. }}\left(a ;(a+2)^{a-4},(a+3)^{4} ;(a+3)^{2}\right)$ contains a nonempty irreducible subset which consists of all the points corresponding to nonsingular irreducible curves $X$ with $B(X)=\left(a ;(a+2)^{a-3}\right.$, 
$\left.(a+3)^{3} ; a+4\right), \mathrm{H}_{*}^{1}\left(\mathscr{I}_{X}\right) \cong M[-a]$.

2) $\overline{\mathrm{H}}\left(a+1 ; a+1,(a+2)^{3},(a+3)^{a-3}\right) \cap \overline{\mathrm{H}}_{\text {а. в. }}\left(a ;(a+3)^{a} ;(a+3)^{2}\right)$

contains a nonempty irreducible subset which consists of all the points corresponding to nonsingular irreducible curves $X$ with $B(X)=(a ; a+2$, $\left.(a+3)^{a-1} ; a+4\right), \mathrm{H}_{*}^{1}\left(\mathscr{I}_{X}\right) \cong M[-a]$.

\section{§3. Nonsingular Irreducible Arithmetically Buchsbaum Curves with Basic Sequence $\left(a ;(a+2)^{a-1}, a+3 ; a+2\right)$}

Let $\mathscr{N}$ be a null correlation bundle, i. e. the vector bundle of rank two obtained as the cohomology of the monad

$$
0 \longrightarrow \mathcal{O}_{\mathbf{P}^{3}}(-1) \stackrel{{ }^{t}\left(-x_{2}, x_{1},-x_{4}, x_{3}\right)}{\longrightarrow}\left(\mathcal{O}_{\mathbf{P}^{3}}\right) \stackrel{\oplus 4}{ } \stackrel{\left(x_{1}, x_{2}, x_{3}, x_{4}\right)}{\longrightarrow} \mathcal{O}_{\mathbf{P}^{3}}(1) \longrightarrow 0 .
$$

For every integer $a \geqq 2, \mathscr{N}(a-1)$ is generated by its global sections and the subscheme of $\mathrm{P}^{3}$ defined as the zero locus of a general section of $\mathscr{N}(a-1)$ is nonsingular by [7; Theorem (3.3)], if $a \geqq 3$. Let $C$ be the curve thus obtained. One sees by the exact sequence

$$
0 \longrightarrow \mathrm{O}_{\mathbf{p}^{3}} \longrightarrow \mathcal{N}(a-1) \longrightarrow \mathscr{I}_{c}(2 a-2) \longrightarrow 0
$$

that $\mathrm{H}_{*}^{1}\left(\mathscr{I}_{c}\right) \cong \mathrm{H}_{*}^{1}(\mathscr{N}(-a+1)) \cong k[-(a-2)]$ and that $h^{0}\left(\mathscr{I}_{c}(\nu)\right)=0$ for $\nu<a$,

$$
h^{0}\left(\mathscr{I}_{c}(\nu)\right)=4\left(\begin{array}{c}
\nu-a+4 \\
3
\end{array}\right)-\left(\begin{array}{c}
\nu-a+5 \\
3
\end{array}\right)-\left(\begin{array}{c}
\nu-a+3 \\
3
\end{array}\right)
$$

for $a \leqq \nu<2 n-2$, from which follows $B(C)=\left(a ; a^{3}, a+1, a+2, \ldots, 2 a-3\right.$; a). When $a=4$, we get in this way a nonsingular irreducible a. B. curve $C$ with $B(C)=\left(4 ; 4^{3}, 5 ; 4\right)$. The following fact can be proved without difficulty as in Proposition 2.3.

Proposition 3.1. Let $a$ be an integer larger than or equal to 4, and suppose a nonsingular irreducible a. B. curve $X$ with $B(X)=\left(a ; a^{a-1}, a+1 ; a\right)$ exists.

1) Let $Y_{1}$ be the curve linked to $X$ by the two surfaces defined respectively by a general element of $I_{X, a}$ and of $I_{X, a+1}$. Then $Y_{1}$ is a nonsingular irreducible a. B. curve with $B\left(Y_{1}\right)=\left(a-1 ; a^{a-3},(a+1)^{2} ; a+1\right)$.

2) Let $Z_{1}$ be the curve linked to $Y_{1}$ by the two surfaces both defined by a general element of $I_{Y_{1}, a+1}$. Then $Z_{1}$ is a nonsingular irreducible a. B. curve with $B\left(Z_{1}\right)=\left(a+1 ;(a+1)^{a}, a+2 ; a+1\right)$. 
3) Let $Y_{2}$ be the curve linked to $X$ by the two surfaces defined respectively by a general element of $I_{X, a}$ and of $I_{X, a+2}$. Then $Y_{2}$ is a nonsingular irreducible a. B. curve with $B\left(Y_{2}\right)=\left(a ; a,(a+1)^{a-3},(a+2)^{2} ; a+2\right)$.

4) Let $Z_{2}$ be the curve linked to $Y_{2}$ by the two surfaces defined respectively by a general element of $I_{Y_{2}, a}$ and of $I_{Y_{2}, a+4^{*}}$. Then $Z_{2}$ is a nonsingular irreducible a. B. curve with $B\left(Z_{2}\right)=\left(a ;(a+2)^{a-1}, a+3 ; a+2\right)$.

Note on the proof. It follows from Corollary 1.3 that $I_{X}$ is generated over $R$ by elements of degree $a$.

Starting from the curve $C$ mentioned just before the above proposition, we can therefore get successively a nonsingular irreducible a. B. curve $X$ with $B(X)=\left(a ;(a+2)^{a-1}, a+3 ; a+2\right)$ for every $a \geqq 4$. These curves also give reducible singular points of $\operatorname{red}\left(\operatorname{Hilb}\left(\mathbf{P}^{3}\right)\right)$.

Proposition 3.2. Let $a$ be an integer larger than or equal to 4 . Then $\overline{\mathrm{H}}\left(a ;(a+2)^{a}\right) \cap \overline{\mathrm{H}}\left(a+1 ;(a+1)^{3},(a+2)^{a-3}, a+3\right)$ is not empty and contains the irreducible subset which consists of the points corresponding to nonsingular irreducible a. B. curves with basic sequence $\left(a ;(a+2)^{a-1}, a+3 ; a+2\right)$.

Proof. Let $X$ be a nonsingular irreducible a. B. curve with $B(X)$ $=\left(a ;(a+2)^{a-1}, a+3 ; a+2\right) . \quad X$ can be deformed in two directions as follows. First of all, since $B(X)$ satisfies the condition of [2; Lemma 5.6], the graded ring $R / I_{X}$ can be flatly deformed into a CohenMacaulay graded ring $R / I$, where $I$ is a homogeneous ideal such that $\operatorname{dim}_{k} I_{\nu}=\operatorname{dim}_{k} I_{X, \nu}$ for $\nu \geqq 0$ (see the proof of the lemma cited). The basic sequence of $I$ turns out to be $\left(a ;(a+2)^{a}\right)$ and this gives one direction of the deformation. On the other hand, the condition of [2; Lemma 5.5] is satisfied as well, so that one obtains a flat deformation of $X$ into a projectively Cohen-Macaulay curve whose basic sequence proves to be $\left(a+1 ;(a+1)^{3},(a+2)^{a-3}, a+3\right)$ (see the proof of the lemma cited), which gives another direction. One sees in fact that $X$ can be deformed in these two ways only.

Q. E. D. 


\section{Appendix : Connection between Multisecants and Basic Sequences}

Let $X$ be a curve in $\mathbb{P}^{3}$ and let $Z$ be another curve the support of which is a line $L=\operatorname{Proj} R /\left(x_{1}-l_{1}, x_{2}-l_{2}\right)$, where $l_{1}, l_{2} \in k(2)_{1}$ for a general choice of coordinates, and suppose $\operatorname{supp}(X \cap L)$ consists of finite points. Suppose further that $\mathcal{O}_{2}$ has a filtration as an $\mathcal{O}_{\mathbf{P}^{3}}$ module of the following form (trivial, if $Z=L$ ):

$$
\begin{aligned}
0 \longrightarrow \mathcal{O}_{L}\left(s_{1}\right) \stackrel{\alpha_{1}}{\longrightarrow} \mathcal{O}_{z_{0}} \stackrel{\beta_{1}}{\longrightarrow} \mathcal{O}_{z_{1}} \longrightarrow 0 \\
0 \longrightarrow \mathcal{O}_{L}\left(s_{2}\right) \stackrel{\alpha_{2}}{\longrightarrow} \mathcal{O}_{z_{1}} \stackrel{\beta_{2}}{\longrightarrow} \mathcal{O}_{z_{2}} \longrightarrow 0 \\
\quad \cdots \ldots \ldots \ldots \ldots \ldots \ldots \ldots \ldots \ldots \ldots \ldots \ldots \ldots \ldots \ldots \ldots \ldots \ldots \ldots \ldots \ldots \ldots \ldots \\
0 \longrightarrow \mathcal{O}_{L}\left(s_{r-1}\right) \stackrel{\alpha_{r-1}}{\longrightarrow} \mathcal{O}_{z_{r-2}} \stackrel{\beta_{r-1}}{\longrightarrow} \mathcal{O}_{z_{r-1}} \longrightarrow 0 \\
0 \longrightarrow \mathcal{O}_{L}\left(s_{r}\right) \stackrel{\alpha_{r}}{\longrightarrow} \mathcal{O}_{z_{r-1}} \stackrel{\beta_{r}}{\longrightarrow} \mathcal{O}_{z_{r}} \longrightarrow 0
\end{aligned}
$$

where $Z_{0}=Z, Z_{r}=L$ and $s_{1} \geqq s_{2} \geqq \ldots \geqq s_{r} \geqq 0$. Put $Y=X \cup Z, t=h^{0}\left(\mathcal{O}_{x_{n}}\right)$ and $c=c(Y):=\max \left\{\nu \mid \mathrm{H}^{1}\left(\mathbf{P}^{3}, \mathscr{I}_{Y}(\nu)\right) \neq 0\right\}$. Let $\left(a ; n_{1}, \ldots, n_{a} ; n_{a+1}, \ldots\right.$, $\left.n_{a+b}\right)$ be the basic sequence of $Y$ and $f_{0}, f_{1}, \ldots, f_{a}, f_{a+1}, \ldots, f_{a+b}$ the generators of $I_{Y}$ associated with it such that

$$
I_{Y}=f_{0} k(0) \oplus \bigoplus_{i=1}^{a} f_{i} k(1) \oplus \bigoplus_{j=1}^{b} f_{a+j} k(2), R=I_{Y} \oplus N_{I_{Y}}
$$

in the notation of [2; Proposition 1.3].

Proposition A.1. In this situation, if $h^{0}\left(\mathcal{O}_{X \cap Z}\right)=(r+1) t$ and $t \geqq t-s_{r} \geqq \ldots \geqq t-s_{1}>c$, then there exist homogeneous polynomials $f_{a+b+1}, \ldots$, $f_{a+b+r+1}$ of degrees $t-s_{1}, t-s_{2}, \ldots, t-s_{r}, t$ respectively such that

$$
I_{X}=f_{0} k(0) \oplus \bigoplus_{i=1}^{a} f_{i} k(1) \bigoplus_{j=1}^{b+r+1} f_{a+j} k(2) .
$$

Proof. Put $Y_{i}=X \cup Z_{i}$. The ideal sheaf of $Y_{i}$ is $\mathscr{I}_{X} \cap \mathscr{I}_{Z_{i}}$ by definition and we have a natural injection

$$
\text { : : } \mathscr{I}_{X} / \mathscr{I}_{Y_{i}} \longrightarrow \mathcal{O}_{z_{i}}
$$

for each $0 \leqq i \leqq r$. Let $\mathscr{L}=\mathcal{O}_{L}(-t)$ denote the ideal sheaf of $X \cap L$ on $L$. One finds from the third row of the following commutative 
diagrams

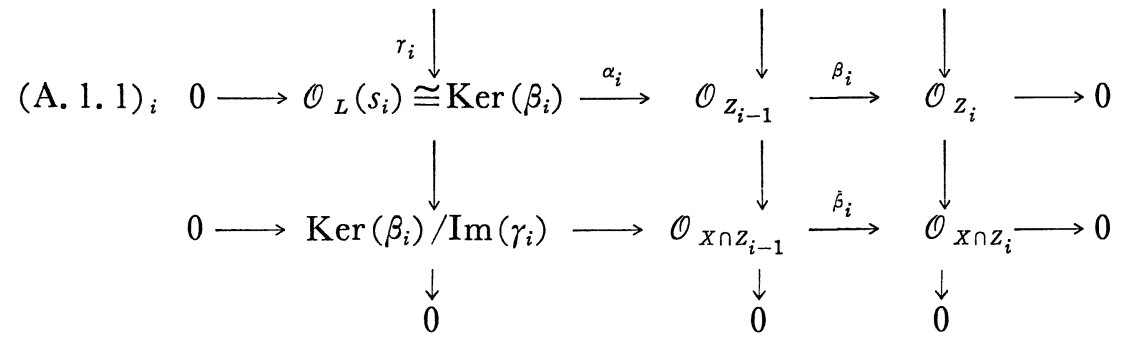

$(1 \leqq i \leqq r)$ with exact rows and columns that

$$
h^{0}\left(\mathcal{O}_{X \cap Z}\right)=\sum_{i=1}^{r} h^{0}\left(\operatorname{Ker}\left(\beta_{i}\right) / \operatorname{Im}\left(\gamma_{i}\right)\right)+h^{0}\left(\mathcal{O}_{X \cap L}\right) .
$$

On the other hand, since $\mathscr{L} \otimes_{\mathcal{O}_{L}} \mathcal{O}_{L}\left(s_{i}\right)=\mathscr{L} \cdot \operatorname{Ker}\left(\beta_{i}\right) \subset \operatorname{Im}\left(\gamma_{i}\right)$, we have $\operatorname{Im}\left(\gamma_{i}\right) \cong \mathcal{O}_{L}\left(s_{i}-t_{i}\right)$ with $t_{i} \leqq t$, so that $h^{0}\left(\operatorname{Ker}\left(\beta_{i}\right) / \operatorname{Im}\left(\gamma_{i}\right)\right)=h^{0}\left(\mathcal{O}_{L}\left(s_{i}\right)\right.$ $\left./ \mathcal{O}_{L}\left(s_{i}-t_{i}\right)\right)=t_{i}$ and $h^{0}\left(\mathcal{O}_{X \cap Z}\right)=\sum_{i=1}^{r} t_{i}+t$. This implies $t_{i}=t$ for all $1 \leqq i \leqq r$ by hypotheses and we obtain a series of exact sequences from the first rows of (A. 1.1) ${ }_{i}$ :

$$
\begin{aligned}
0 \longrightarrow \mathcal{O}_{L}\left(s_{1}-t\right) \stackrel{\alpha_{1}^{\prime}}{\longrightarrow} \mathscr{I}_{X} / \mathscr{I}_{Y} \stackrel{\beta_{1}^{\prime}}{\longrightarrow} \mathscr{I}_{X} / \mathscr{I}_{Y_{1}} \longrightarrow 0 \\
0 \longrightarrow \mathcal{O}_{L}\left(s_{2}-t\right) \stackrel{\alpha_{2}^{\prime}}{\longrightarrow} \mathscr{I}_{X} / \mathscr{I}_{Y_{1}} \stackrel{\beta_{2}^{\prime}}{\longrightarrow} \mathscr{I}_{X} / \mathscr{I}_{Y_{2}} \longrightarrow 0 \\
\ldots \ldots \ldots \ldots \ldots \ldots \ldots \ldots \ldots \ldots \ldots \ldots \ldots \ldots \ldots \ldots \ldots \ldots \ldots \ldots \ldots \\
0 \longrightarrow \mathcal{O}_{L}\left(s_{r-1}-t\right) \stackrel{\alpha_{r-1}^{\prime}}{\longrightarrow} \mathscr{I}_{X} / \mathscr{I}_{Y_{r-2}} \stackrel{\beta_{r-1}^{\prime}}{\longrightarrow} \mathscr{I}_{X} / \mathscr{I}_{Y_{r-1}} \longrightarrow 0 \\
0 \longrightarrow \mathcal{O}_{L}\left(s_{r}-t\right) \stackrel{\alpha_{r}^{\prime}}{\longrightarrow} \mathscr{I}_{X} / \mathscr{I}_{Y_{r-1}} \stackrel{\beta_{r}^{\prime}}{\longrightarrow} \mathscr{I}_{X} / \mathscr{I}_{Y_{r}} \cong \mathcal{O}_{L}(-t) \longrightarrow 0 .
\end{aligned}
$$

Consider $\mathcal{O}_{z_{i}}(0 \leqq i \leqq r)$ as $\mathcal{O}_{L}$-modules through the natural injection $k(2) \subset R$. All these sequences then split as $\mathcal{O}_{L}$-modules, namely there exist $\mathcal{O}_{L}$-module homomorphisms

$$
\varepsilon_{i}: \mathcal{O}_{L}\left(s_{i}-t\right) \longrightarrow \mathscr{I}_{X} / \mathscr{I}_{Y} \quad(1 \leqq i \leqq r+1)
$$

which give rise to an isomorphism

$$
\varepsilon:=\sum_{i=1}^{r+1} \varepsilon_{i}: \bigoplus_{i=1}^{r+1} \mathcal{O}_{L}\left(s_{i}-t\right) \longrightarrow \mathscr{I}_{X} / \mathscr{I}_{Y}
$$


where $\varepsilon_{1}=\alpha_{1}^{\prime}$ and we have put $s_{r+1}=0$ for convenience sake. In the diagram

$$
\begin{aligned}
0 \longrightarrow \mathscr{I}_{Y}(\nu) \longrightarrow \mathscr{I}_{X}(\nu) \longrightarrow & \mathscr{I}_{X} / \mathscr{I}_{Y}(\nu) \longrightarrow 0 \\
& \varepsilon ? \uparrow \text { (isomo phism as } \mathcal{O}_{L}-\text { modules) } \\
& \bigoplus_{i=1}^{r+1} \mathcal{O}_{L}\left(s_{i}-t+\nu\right)
\end{aligned}
$$

let $\bar{f}_{a+b+i} \in \mathrm{H}^{0}\left(\mathscr{I}_{X} / \mathscr{I}_{Y}\left(t-s_{i}\right)\right)$ be the image of $1 \in \mathrm{H}^{0}\left(\mathcal{O}_{L}\right)$ under $\varepsilon_{i}(1 \leqq i \leqq r+1)$. Since $\mathrm{H}^{1}\left(\mathscr{I}_{Y}(\nu)\right)=0$ for $\nu \geqq t-s_{1}$ by hypotheses, there exists a homogeneous polynomial $f_{a+b+i} \in \mathrm{H}^{0}\left(\mathscr{I}_{X}\left(t-s_{i}\right)\right)$ contained in $N_{I_{Y}}$ such that $f_{a+b+i}\left(\bmod I_{Y}\right)=\bar{f}_{a+b+i}$ for every $i$. Besides, we have $\mathrm{H}^{0}\left(\mathscr{I}_{X} / \mathscr{I}_{Y}(\nu)\right)=0 \quad$ for $\nu \leqq t-s_{1}-1$, therefore $I_{X}=I_{Y} \oplus \bigoplus_{j=1}^{r+1} f_{a+b+j} k(2)$, which proves our assertion.

Q. E. D.

Corollary A.2. With the notation above, we have

$$
\left(x_{j}-l_{j}\right) \operatorname{Im}\left(\varepsilon_{i}\right) \subset \operatorname{Im}\left(\sum_{u=1}^{i-1} \varepsilon_{u}\right)
$$

and

$$
\left(x_{j}-l_{j}\right) \operatorname{Im}\left(\varepsilon_{1}\right)=0 \quad \text { for } j=1,2 .
$$

Proof. Each $\varepsilon_{i}$ satisfies $\beta_{i-1}^{\prime} \circ \cdots \circ \beta_{1}^{\prime} \circ \varepsilon_{i}=\alpha_{i}^{\prime}$ for $2 \leqq i \leqq r$ and $\beta_{r}^{\prime} \circ \cdots \circ \beta_{1}^{\prime}$ ${ }^{\circ} \varepsilon_{r+1}=i d$. Therefore

$$
\begin{aligned}
\left(\beta_{q}^{\prime} \circ \cdots \circ \beta_{1}^{\prime}\right)\left(\left(x_{j}-l_{j}\right) \varepsilon_{i}(g)\right) & =\left(x_{j}-l_{j}\right)\left\{\left(\beta_{q}^{\prime} \circ \cdots \circ \beta_{1}^{\prime}\right) \circ \varepsilon_{i}(g)\right\} \\
& =\beta_{q}^{\prime} \circ \cdots \circ \beta_{i}^{\prime} \circ \alpha_{i}^{\prime}\left(\left(x_{j}-l_{j}\right) g\right)=0
\end{aligned}
$$

for $g \in \mathcal{O}_{L}\left(s_{i}-t\right), q \geqq i-1, j=1,2$. Suppose $h \in \operatorname{Im}\left(\varepsilon_{i}\right)$ and $\left(x_{j}-l_{j}\right) h$ $=\sum_{u=1}^{r+1} \varepsilon_{u}\left(h_{u j}\right)(j=1,2)$. One sees first $0=\left(\beta_{r}^{\prime} \circ \cdots \circ \beta_{1}^{\prime}\right)\left(\left(x_{j}-l_{j}\right) h\right)=\left(\beta_{r}^{\prime} \circ \cdots \circ \beta_{1}^{\prime}\right)$ $\left(\sum_{u=1}^{r+1} \varepsilon_{u}\left(h_{u j}\right)\right)=h_{r+1, j}$, in the second place $0=\left(\beta_{r-1}^{\prime} \circ \cdots \circ \beta_{1}^{\prime}\right)\left(\left(x_{j}-l_{j}\right) h\right)$ $=\left(\beta_{r-1}^{\prime} \circ \cdots \circ \beta_{1}^{\prime}\right)\left(\sum_{u=1}^{r} \varepsilon_{u}\left(h_{u j}\right)\right)=\alpha_{r}^{\prime}\left(h_{r j}\right)$, that is $h_{r j}=0$, and by induction finally $\begin{array}{ll}\text { finds } h_{i j}=h_{i+1, j}=\cdots=h_{r+1, j}=0 . & \text { Q. E. D. }\end{array}$

Corollary A.3. The notation being as in Proposition A. 1, let

$$
\lambda_{2}=\left[\begin{array}{lll}
U_{01} & U_{02} & 0 \\
U_{1} & U_{2} & U_{4} \\
U_{21} & U_{3} & U_{5}
\end{array}\right] \quad\left(r e s p . \quad \lambda_{2}^{\prime}=\left[\begin{array}{ccc}
U_{01}^{\prime} & U_{02}^{\prime} & 0 \\
U_{1}^{\prime} & U_{2}^{\prime} & U_{4}^{\prime} \\
U_{21}^{\prime} & U_{3}^{\prime} & U_{5}^{\prime}
\end{array}\right]\right)
$$

be the matrix of relations among $f_{0}, \ldots, f_{a+b}$ (resp. $\left.f_{0}, \ldots, f_{a+b+r+1}\right)$ computed 
by [1; Theorem 1.6]. Then

$$
\begin{aligned}
& U_{01}^{\prime}=U_{01}, U_{1}^{\prime}=U_{1}, U_{21}^{\prime}=\left[\begin{array}{c}
U_{21} \\
0
\end{array}\right], \\
& U_{3}^{\prime}=\left[\begin{array}{cccc}
U_{3} & & * & \\
& x_{1}-l_{1} & & \\
0 & 0 & \ddots & * \\
& & & x_{1}-l_{1}
\end{array}\right] \\
& \text { and } U_{5}^{\prime}=\left[\begin{array}{cccc}
U_{5} & & * & \\
& x_{2}-l_{2} & \\
0 & 0 & \ddots & * \\
0 & 0 & & x_{2}-l_{2}
\end{array}\right] .
\end{aligned}
$$

If the condition $t-s_{i} \geqq n_{a}(1 \leqq i \leqq r+1)$ is fulfilled additionaly, then the basic sequence of $X$ is $\left(a ; n_{1}, \ldots, n_{a} ; n_{a+1}, \ldots, n_{a+b}, t-s_{1}, t-s_{2}, \ldots, t-s_{r}, t\right) u p$ to a permutation of $n_{a+1}, \ldots, n_{a+b}, t-s_{1}, t-s_{2}, \ldots, t-s_{r}, t$.

Proof. The first part is clear. To prove the second part, notice first of all that the entries of $U_{4}^{\prime}$ are in $\left(x_{3}, x_{4}\right) k(2)$, if $t-s_{i} \geqq n_{a}$. One sees then by the formula

$$
f_{i}=(-1)^{i} \operatorname{det} W_{2}^{\prime}(i) / \operatorname{det} U_{5}^{\prime}\left(W_{2}^{\prime}=\lambda_{2}^{\prime}(a+1, \ldots, a+b+r+1)\right)
$$

that $f_{a+j} \equiv 0\left(\bmod \left(x_{3}, x_{4}\right) R\right)$ for $1 \leqq j \leqq b+r+1$ and $I_{Y} \equiv I_{X}\left(\bmod \left(x_{3}, x_{4}\right) R\right)$. Since the coordinates were chosen sufficiently generally from the first, we find $B(X)=\left(a ; n_{1}, \ldots, n_{a} ; \bar{n}^{\prime 2}\right)$ for some increasing sequence of integers $n^{\prime 2}$ and this must be equal to $\left(n_{a+1}, \ldots, n_{a+b}, t-s_{1}, t-s_{2}, \ldots\right.$, $\left.t-s_{r}, t\right)$ up to a permutation by the definition of the basic sequence.

Q. E. D.

Example A. 4. Let $X$ be an integral curve belonging to the linear system $\left|14 l-5 e_{1}-4\left(e_{2}+e_{3}\right)-3\left(e_{4}+e_{5}+e_{6}\right)\right|$ on a smooth cubic surface $S$ and let $G_{i} \in\left|2 l-\sum_{j \neq i} e_{j}\right|$ be the line for $1 \leqq i \leqq 6$. Set $X_{1}=X+2 G_{1}$, $X_{2}=X_{1}+G_{2}$ and $X_{3}=X_{2}+G_{3}$. Then $\left(2 G_{1}\right) \cdot X=22, G_{1} \cdot X=11, G_{2} \bullet X_{1}=10$, $G_{3} \cdot X_{2}=10$ and $\mathcal{O}_{2 G_{1}}$ has the filtration

$$
\left.0 \longrightarrow \mathcal{O}_{G_{1}}(1) \cong \mathcal{O}_{S}\left(-G_{1}\right)\right|_{G_{1}} \longrightarrow \mathcal{O}_{2 G_{1}} \longrightarrow \mathcal{O}_{G_{1}} \longrightarrow 0 \text {. }
$$

One can check without difficulty that $X_{3} \sim 22 l-7 \sum_{i=1}^{6} e_{i}$ is projectively Cohen-Macaulay, namely $h^{1}\left(\mathscr{I}_{X_{3}}(\nu)\right)=h^{1}\left(\mathcal{O}_{S}\left(-X_{3}+\nu h\right)\right)=0(h$ deno- 
ting a hyperplane section) for all integers $\nu$, and finds $B\left(X_{3}\right)=\left(3 ; 9^{3}\right)$. Now, apply Corollary A. 3 successively starting with the curve $X_{3}$. Then we get $B\left(X_{2}\right)=\left(3 ; 9^{3} ; 10\right), B\left(X_{1}\right)=\left(3 ; 9^{3} ; 10^{2}\right)$ and finally $B(X)$ $=\left(3 ; 9^{3} ; 10^{3}, 11\right)$.

Example A. 5. Let $\tilde{S_{0}} \subset \mathbb{P}^{4}$ be the cone over a nonsingular rational curve of degree $3, \pi: S_{0} \longrightarrow \tilde{S}_{0}$ the blowing up of $\tilde{S}_{0}$ with center its vertex and let $h, r$ be the pullback of a hyperplane section and the line of ruling respectively. We consider a nonsingular irreducible curve $X$ in $\mathbf{P}^{3}$ obtained by projecting a smooth curve $C$ on $\tilde{S}_{0}$ whose strict transform $\hat{C}$ belongs to the linear system $|m h+r|$ from a general point not on $\tilde{S}_{0}$, where $m$ is an integer larger than 2. $X$ lies on a cubic surface $S_{0}$ with a double line $L$ which is in fact a cone over a singular rational curve of degree 3 in $\mathbb{P}^{2}$ with a node. Denote the projection by $p: \tilde{S_{0}} \longrightarrow S_{0}$ and put $\hat{p}=p \circ \pi$. We see $\hat{p}^{-1}(L)$ is the union of two different lines of ruling $r_{1}, r_{2}$ and of the exceptional divisor $E$ of $\hat{S}_{0}$, and we may assume $\hat{C} \cap E \cap\left\{r_{1}, r_{2}\right\}=\phi$. The curve $X$ and $L$ therefore intersect quasi-transversally at $s_{0}=\hat{p}(E)$ and $h^{0}\left(\mathcal{O}_{X \cap L}\right)$ $=\left(r_{1}+r_{2}\right) \cdot \hat{C}+1=2 m+1$. Set $Y=X \cup L$ and $\overline{\mathscr{I}}_{Y}=\mathscr{I}_{Y /} / \mathscr{I}_{S_{0}}$. Since $Y$

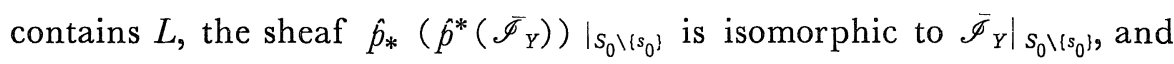
since $\left\{s_{0}\right\}$ is of codimension 3 in $\mathbb{P}^{3}$, it follows from the sequence $0 \longrightarrow \mathcal{O}_{\mathrm{p}^{3}}(-3) \longrightarrow \mathscr{I}_{Y} \longrightarrow \overline{\mathscr{I}}_{Y} \longrightarrow 0$ that this isomorphism can be extended to the whole of $S_{0}$, i. e. $\hat{p}_{*}\left(\hat{p}^{*}\left(\overline{\mathscr{I}}_{Y}\right)\right)=\overline{\mathscr{I}}_{Y_{0}}$ In addition, we have $\hat{p}^{*}\left(\overline{\mathscr{I}}_{Y}\right)=\mathcal{O}_{\hat{s}_{0}}\left(-\hat{C}-E-r_{1}-r_{2}\right)=\mathcal{O}_{\hat{s}_{0}}(-(m+1) h)$, so we find by the spectral sequence that $\mathrm{H}_{*}^{1}\left(\mathscr{I}_{Y}\right) \cong \mathrm{H}_{*}^{1}\left(\dot{\mathscr{J}}_{Y}\right)=0 . \quad Y$ is thus projectively Cohen-Macaulay and after a simple computation we get $B(Y)$ $=\left(3 ; m+1,(m+2)^{2}\right)$, whence $B(X)=\left(3 ; m+1,(m+2)^{2} ; 2 m+1\right)$.

Let $\tilde{S}$ be a nonsingular rational scroll of degree 3 in $\mathbb{P}^{4}, p: \tilde{S} \longrightarrow$ $S \subset \mathbb{P}^{3}$ a projection from a general point not on $\tilde{S}$ and let $h, r \subset \tilde{S}$ again denote the hyperplane section and the line of ruling respectively. Then we find analogously $B\left(p\left(G_{1}\right)\right)=\left(3 ; m+1,(m+2)^{2} ; 2 m+1\right)$ (the same as above) for a nonsingular irreducible curve $C_{1}$ belonging to the linear system $|m h+r|$ and $B\left(p\left(C_{2}\right)\right)=\left(3 ;(m+2)^{3} ; m+2,2 m\right)$ for $C_{2}$ belonging to $|(n+1) h-2 r|$. 


\section{References}

[1] Amasaki, M., Preparatory Structure Theorem for Ideals Defining Space Curves, Publ. RIMS, Kyoto Univ., 19 (1983), 493-518.

[2] On the Structure of Arithmetically Buchsbaum Curves in $\mathbf{P}_{k}^{3}$, Publ. RIMS, Kyoto Univ., 20 (1984), 793-837.

[3] Eisenbud, D. and Goto, S., Linear Free Resolutions and Minimal Multiplicity, Journal of Algebra, 88 (1984), 89-133.

[4] Gruson, L. et Peskine, C., Genre des courbes de l'espace projectif, Proceedings, Troms $\phi$ Norway 1977, Lecture Notes in Mathematics No. 687, Springer-Verlag.

[5] Genre des courbes de l'espace projectif (II), Ann. Scient. Ec. Norm. Sup., $4^{a}$ série, t. 15 (1982), 401-418.

[6] Harris, J., Curves in Projective Space, Les Presses de l'Université de Montréal, 1982.

[7] Kleimann, S. L., Geometry on Grassmannians and Application to Splitting Bundles and Smoothing Cycles, Publ. Math. I.H.E.S., No. 36 (1969), 281-297.

[8] Peskine, C. et Szpiro, L., Liaison des variétés algebriques I, Inventiones math., 26 (1974), 271-302.

[9] Rao, A. P., Liaison among Curves in $\mathbf{P}^{3}$, Inventiones math., 50 (1979), 205-217. 